\title{
Evaluation of AIRS Cloud Phase Classification over the Arctic Ocean against Combined CloudSat-CALIPSO Observations
}

\author{
Colten A. Peterson \\ Department of Climate and Space Sciences and Engineering, University of Michigan, Ann Arbor, Michigan \\ QING Yue, BRIAN H. KAHN, AND ERIC FETZER \\ Jet Propulsion Laboratory, California Institute of Technology, Pasadena, California \\ Xianglei HuANG \\ Department of Climate and Space Sciences and Engineering, University of Michigan, Ann Arbor, Michigan
}

(Manuscript received 16 January 2020, in final form 27 April 2020)

\begin{abstract}
Cloud phase retrievals from the Atmospheric Infrared Sounder (AIRS) are evaluated against combined CloudSat-CALIPSO (CCL) observations using four years of data (2007-10) over the Arctic Ocean. AIRS cloud phase is evaluated over sea ice and open ocean separately using collocated CCL and AIRS fields of view (FOVs). In addition, AIRS and CCL cloud phase occurrences are evaluated seasonally, zonally, and with respect to total column water vapor (TCWV) and the temperature difference between 1000 and $300 \mathrm{hPa}\left(\Delta T_{1000-300}\right)$. Last, collocated MODIS cloud information is implemented in a 1-month case study to assess the relationship between AIRS and CCL phase decisions, cloud cover, and cloud phase throughout the AIRS FOV. Depending on the surface type, AIRS classification skill for single-layer ice and liquid-phase clouds is over the ranges of $85 \%-95 \%$ and $22 \%-32 \%$, respectively. Most unknown and liquid AIRS phase classifications correspond to mixed-phase clouds. AIRS ice-phase relative occurrence is biased low relative to CCL. However, the liquid-phase relative occurrence is similar between the two instruments. When compared with the CCL climatology, AIRS accurately represents the seasonal cycle of liquid and ice cloud phase across the Arctic as well as the relationship between cloud phase and TCWV and $\Delta T_{1000-300}$ regime in some cases. The more heterogeneous the MODIS cloud macrophysical properties within an AIRS FOV are, the more likely it is that the AIRS FOV is classified as unknown phase.
\end{abstract}

\section{Introduction}

The Arctic is an important component of the global climate system, and changes in this region can have farreaching impacts. Recent trends in surface-air warming (e.g., Boisvert and Stroeve 2015; Peterson et al. 2019) and sea ice decline (Cavalieri and Parkinson 2012) indicate that knowledge of the Arctic surface energy budget is critical for understanding future changes in the region. The radiative effects of clouds can modify the surface climate in the Arctic, particularly through infrared (IR) warming that occurs during most of the year (Intrieri et al. 2002). In the summertime, clouds modulate the amount of solar radiation absorbed by the Arctic

Corresponding author: Colten A. Peterson, coltenp@umich.edu
Ocean (Kay and L'Ecuyer 2013). The phase of a cloud, that is, whether it is composed of liquid droplets, ice particles, or both, plays a critical role in the radiative impact of the cloud on surface climate. Liquid clouds tend to have a higher opacity and contribute more to IR warming of the surface than ice clouds (Shupe and Intrieri 2004; Cesana et al. 2012). Moreover, liquid droplets tend to be smaller than ice particles, and thus the cloud albedo can also be strongly impacted by cloud phase (Shupe and Intrieri 2004).

Arctic clouds are unique as compared with those in lower latitudes, because mixed-phase clouds are ubiquitous in the region and can exist at temperatures as cold as $-40^{\circ} \mathrm{C}$ (Shupe 2011). Thus, clouds containing liquid water can often exist during the Arctic winter. The complexity of Arctic cloud microphysics and a lack of 
understanding of the connections between Arctic cloud phase and other geophysical parameters have proved to be a challenge for realistically representing Arctic clouds in coupled climate model simulations. For example, Cesana et al. (2012) showed that the underestimation of liquid cloud occurrence in two climate models, particularly in the Arctic autumn and winter, can lead to an underestimation of the frequency of occurrence of "radiatively opaque" states in which downwelling longwave radiation from liquid containing clouds nearly balances the upwelling longwave emission from the surface. This can lead to an overestimation of "radiatively clear" states in which the net surface longwave emission can be $50-100 \mathrm{~W} \mathrm{~m}^{-2}$ larger than the downwelling longwave emission from the atmosphere, resulting in rapid surface cooling.

Because it is extremely challenging to maintain longterm ground observation sites in the harsh Arctic weather, satellite observations are critically needed for the understanding of connections between Arctic cloud phase and other surface and atmospheric parameters. Numerous cloud phase algorithms exist for spaceborne instruments such as AVHRR (Key and Intrieri 2000), POLDER (Goloub et al. 2000; Riedi et al. 2010), Cloud-Aerosol Lidar and Infrared Pathfinder Satellite Observations (CALIPSO; Hu et al. 2009), MODIS (Baum et al. 2012; Marchant et al. 2016), and the Atmospheric Infrared Sounder (AIRS; Kahn et al. 2014). The A-Train constellation includes a suite of instruments whose measurements can be collocated, and this presents a valuable opportunity to use multiple observations to study Arctic cloud phase and to evaluate the cloud phase algorithm designed for each individual instrument. Instruments like the CALIPSO lidar (which was present in the A-Train from 2006 to 2011) provide vertical profiles of cloud phase using lidar backscatter and depolarization (Hu et al. 2009). CloudSat, a 94-GHz cloud profiling radar, orbits Earth together with CALIPSO, and the combination of CloudSat radar and CALIPSO lidar has also been used to estimate cloud phase (Sassen et al. 2008).

While estimating cloud phase with active sensors is considered a benchmark, such estimates are only available along a narrow track (e.g., 90-100-m track width for CALIPSO). Passive A-Train instruments like MODIS and AIRS have larger horizontal fields of view (FOV; 1 and $13.5 \mathrm{~km}$ at nadir, respectively) relative to active sensors, and their cross-track scanning allows for coverage of areas that the active sensors miss during an overpass. Passive cloud phase discrimination relies mainly on the differences between ice particles and liquid droplets in terms of either IR absorption or shortwave reflection, or both of them. Infrared cloud phase methods are fundamentally limited when mixedphase clouds are present (e.g., Baum et al. 2000), which presents a challenge in the Arctic where mixed-phase clouds are ubiquitous. However, because solar illumination is scarce throughout the Arctic year, an IR approach is the only practical option for passively estimating Arctic cloud phase during all seasons.

The MODIS Collection 6 products include both an IR-only (Baum et al. 2012) and IR-shortwave (Marchant et al. 2016) cloud phase identification at $1-\mathrm{km}$ spatial resolution. AIRS, which estimates cloud phase with IR radiances only (Kahn et al. 2014), differs from MODIS in that it has coarser spatial resolution ( $13.5 \mathrm{~km}$ at nadir) but much finer spectral resolution and more spectral channels. Kahn et al. (2011) showed that AIRS FOVs that contain both cloudy and clear MODIS pixels tended to lead to cloud phase decision errors or unknownphase classifications by AIRS. However, the finer spectral resolution of AIRS allows for a selection of channels that are less impacted by water vapor absorption and are more sensitive to the spectrally dependent differences between liquid and ice extinction of IR radiation; this increases the AIRS sensitivity to cloud phase relative to the MODIS IR-only approach (Nasiri and Kahn 2008). The high spectral resolution and over 17 years of observations from AIRS implies that AIRS could be a useful tool for studying Arctic cloud phase climatology. However, AIRS cloud phase retrievals have not been rigorously evaluated against benchmark active sensors in the Arctic region. Such a study must be undertaken before AIRS cloud phase information can be used for studies of Arctic cloud phase climatology.

Jin and Nasiri (2014, hereinafter JN14) compared AIRS cloud phase classifications to collocated CALIPSO observations on a global scale. Some of the key findings from their study were 1) AIRS cloud phase agrees with CALIPSO cloud phase more for single-layer systems than for multilayer systems, 2) AIRS phase tends to agree more with CALIPSO ice phase than CALIPSO liquid phase, 3) AIRS tends to classify most liquid cloud scenes as unknown phase, and 4) AIRS also tends to classify scenes containing both ice and liquid clouds as unknown phase. These global-scale statistics revealed much about the AIRS phase algorithm's limitations, but performing such an evaluation with a focus on the Arctic region allows for an assessment of AIRS phase classification in a region characterized by markedly different cloud properties, surface conditions, and atmospheric conditions from those at lower latitudes. Water vapor amount is a critical factor for AIRS cloud phase decisions (e.g., Kahn et al. 2011), and the relatively dry Arctic atmosphere may cause AIRS Arctic cloud phase classification skill to be different than the global classification skill. 
Given the lack of knowledge and globally climatic importance of Arctic cloud phase, evaluating AIRS cloud phase retrievals in this region is warranted. Also, such an evaluation can provide information for improving the AIRS cloud phase algorithm, especially in the Arctic.

In this study we systematically evaluate AIRS cloud phase in the Arctic from 2007 to 2010 against active sensor observations, but there are several differences relative to the JN14 approach that make the evaluation of AIRS cloud phase more targeted to the Arctic region:

1) We use CloudSat and CALIPSO (CCL) observations as opposed to CALIPSO only. JN14's mixed-phase category was based on spatial combinations of ice and liquid CALIPSO pixels within the AIRS FOV because CALIPSO cannot detect the coexistence of ice and liquid in a single cloud layer. However, because the CloudSat radar is sensitive to ice particles within liquid layers that the CALIPSO lidar is sensitive to, the use of CCL allows for an evaluation of AIRS phase classification for mixed-phase cloud scenes that exist on smaller spatial scales than CALIPSO can detect, as mixed-phase horizontal scales of tens of meters are common in high latitudes (e.g., Thompson et al. 2018).

2) JN14 characterized AIRS phase classification in the presence of multiple cloud layers but assumed that AIRS was most sensitive to the phase of the uppermost layers and hence did not focus on the underlying cloud layer's phase. This is a valid assumption when the upper cloud layer is optically thick in the IR (e.g., visible optical depths of $\sim 5$ or more), but Arctic clouds can be semitransparent, especially ice clouds, and thus the phase of the underlying cloud layers could influence the AIRS cloud phase classification. Therefore, in this study, we investigate the impact of different combinations of phases in multilayer cloud systems on the AIRS phase classification statistics.

3) JN14 used cloud amount from the narrow CALIPSO track to separate AIRS FOVs into cloud amount categories. In this study, AIRS cloud phase is instead evaluated using "overcast" groupings of CCL FOVs. To further investigate how cloud amount and cloud phase distributions throughout the entire AIRS FOV impact AIRS cloud phase classifications in the Arctic, a case study of collocated MODIS cloud observations is used to investigate the connections between MODIS cloud phase and cloud fraction, CCL phase classification, and AIRS phase classification. The case study allows for an evaluation of the representativeness of CCL cloud phase throughout the AIRS FOV. Like JN14, the cloud phase along the CCL track is assumed to be statistically representative of the cloud phase in the AIRS FOV, which may not always be valid, but is a necessary assumption when evaluating AIRS phase with CCL.

4) Given the unique characteristics of the Arctic climate system, the AIRS phase classification is evaluated over open ocean and sea ice. These surface types can represent opposite extremes in Arctic climate conditions such as atmospheric composition and surface emissivity, and such factors may influence AIRS phase classification skill in the Arctic. The relationship between vertical distributions of CCL cloud phase occurrence and AIRS phase classifications over both surface types is also examined. Last, the AIRS and CCL cloud phase occurrences are compared at different Arctic zonal bands during different seasons and, using a composite analysis, the impacts of temperature difference between 1000 and $300 \mathrm{hPa}\left(\Delta T_{1000-300}\right)$ and total column water vapor (TCWV) on AIRS and CCL cloud phase occurrence over both surface types are examined.

The rest of the paper is organized as follows: section 2 describes the instruments and data used, the collocation methods, and considerations pertaining to CCL cloud amount and layering in the AIRS phase evaluation. Section 3 includes the results and discussions of the AIRS phase evaluations, section 4 discusses the MODIS case study, and section 5 describes the conclusions and suggestions for improving the AIRS cloud phase algorithm with respect to the Arctic.

\section{Data and methods}

\section{a. AIRS and its Level 2 cloud phase algorithm}

The AIRS instrument is an infrared grating spectrometer that is aboard the Aqua satellite in the A-Train constellation. Aqua has a sun synchronous orbit with equatorial crossing times of 0130 and 1330 local time. AIRS is a cross-track scanning instrument with a swath range from $-49^{\circ}$ to $49^{\circ}$ and has FOV of $1.1^{\circ}$, which corresponds to a $13.5-\mathrm{km}$ nadir footprint. AIRS records spectra in 2378 channels in three bands spanning the mid-IR to the near-IR and has a spectral resolving power of $\lambda / d \lambda=1200$ (Aumann et al. 2003; Chahine et al. 2006).

AIRS thermodynamic cloud phase, which is available in the AIRS, version 6 (V6), Level 2 (L2) Support Product, is based on a set of brightness temperature difference tests and thresholds using the channels 960 , 1231, 930, and $1227 \mathrm{~cm}^{-1}$ (Nasiri and Kahn 2008; Kahn et al. 2014). The tests are used to classify the AIRS FOV as containing liquid, ice, or unknown cloud phase. The phase tests are applied only to AIRS FOVs where the 
total two-layer effective cloud fraction $(\mathrm{ECF})$ is $>0.01$. There are four ice tests and two liquid tests. The results of all tests are summed and the classification is ice if the value is positive (from +1 to +4 ), unknown if 0 , and liquid if negative $(-1$ and -2$)$. The larger positive or negative values indicate higher confidence in the phase classification. The individual test results are stored in the field called cloud_phase_bits, and the sum is reported in the field called cloud_phase_3 $\times 3$.

ECF is retrieved after completion of the AIRS V6 cloud clearing steps by comparing observed and computed radiances in a set of channels that are sensitive to cloud amount and height. ECF is the product of cloud amount and cloud emissivity and is retrieved for up to two layers in the AIRS FOV. This is reported in the AIRS L2 Standard Product. The surface characterization for the AIRS FOVs used in the study is based on AMSU retrievals of surface emissivity in the $45 \mathrm{~km}$ AMSU field of regard. The product is called MWSurfType and is an AIRS V6L2 Standard Product. The generalized surface types are unknown, coastline, open ocean, sea ice, nonfrozen land, and frozen land. Only open ocean and sea ice surface types are used in this study.

\section{b. Combined CloudSat-CALIPSO cloud retrievals}

The version R04 2B-CLDCLASS-lidar (CCL; Wang et al. 2013) product is used as the benchmark cloud phase reference. This product uses collocated CloudSat radar reflectively factors and CALIPSO attenuated backscatter coefficients (provided in the lidar-AUX 004 auxiliary CCL product), where up to 15 CALIPSO footprints can lie within a $1.8 \mathrm{~km} \times 1.5 \mathrm{~km}$ CloudSat FOV. CALIPSO attenuated backscatter coefficients are averaged to the CloudSat horizontal resolution and the native $30-\mathrm{m}$ vertical CALIPSO resolution is retained.

Radar and lidar have different sensitivities to cloud properties. The CloudSat $94 \mathrm{GHz}$ radar can penetrate deeper into cloud layers and is sensitive to large ice particles, while the CALIPSO $532 \mathrm{~nm}$ lidar pulse is more sensitive to cirrus clouds and liquid droplets. By synergizing the instruments, CCL can classify up to 10 layers as ice, liquid, or mixed phase. Distinct cloud layers are identified with a separation of $\sim 480 \mathrm{~m}$ (Marchand et al. 2008). In this study, we only use up to 5 layers because higher-layer amounts occur in less than $0.06 \%$ of global CCL observations during our study period of 2007-10 (Wang et al. 2016). The CCL product also provides the number of cloud layers and the cloud base and top heights in each CCL FOV. To avoid potential misidentification of cloudy scenes near the surface, which is called "ground clutter" (Tanelli et al. 2008), we only use CCL cloud layers in which the cloud base lies above $0.5 \mathrm{~km}$.

\section{c. ERA5 total column water vapor and atmospheric temperature}

TCWV and profiles of atmospheric temperature for January 2007-December 2010 were obtained from the European Centre for Medium-Range Weather Forecasts (ECWMF) ERA5 (Hersbach et al. 2018). Data are 1-hourly and at $0.25^{\circ} \times 0.25^{\circ}$ spatial resolution. Profiles of atmospheric temperature are provided at 37 pressure levels from 1000 to $1 \mathrm{hPa}$.

\section{d. MODIS cloud property retrievals}

Aqua MODIS Collection 6 (MYDO6_L2) L2 cloud $1-\mathrm{km}$ daytime only cloud mask (Cloud_Mask_1 km; Platnick et al. 2013) and cloud phase (Cloud_Phase_ Optical_Properties) from the Cloud Optical Properties Product (Marchant et al. 2016; Platnick et al. 2017) are used in the case study described in section 4 . The cloud mask flags a pixel as confidently clear, probably clear, probably cloudy, or confident cloudy. The cloud phase product flags a pixel as ice, liquid, or unknown. Note that the IR-only MODIS thermodynamic cloud phase product is not used at all in this study, and thus only daytime MODIS data are used.

\section{e. AIRS, CCL, and MODIS collocation strategy}

To collocate CCL and AIRS FOVs, collocation indices during the 2007-10 period from the Making Earth System Data Records for Use in Research Environments (MEASURES) Project (Fetzer 2012) were used. A nearest-neighbor approach was used to match CloudSat and AIRS FOVs based on the latitude and longitude pair (Kahn et al. 2008; Yue et al. 2011; Fetzer 2012). Because of the orbital configuration and viewing geometries of the instruments, CloudSat and CALIPSO FOVs can only overlap with near-nadir AIRS FOVs, and thus only such AIRS FOVs are used in this study. Approximately 15-19 CloudSat (and therefore CCL) FOVs lie within a 13.5 -km near-nadir AIRS FOV. The MEASURES collocation indices contain the AIRS granule number, FOV indices, and an indexed array of CCL cloud products. Only AIRS granules falling between $60^{\circ}$ and $90^{\circ} \mathrm{N}$ are used. Since the CCL orbit is limited to $<83^{\circ} \mathrm{N}$, the study area is confined to $60^{\circ}-$ $83^{\circ} \mathrm{N}$. This provides a sample set of $\sim 4$ million nearnadir AIRS FOVs and $\sim 61$ million CCL FOVs over the 4-yr period. To provide an illustration of the AIRSCCL collocation strategy, Fig. 1 shows vertical curtains of CCL cloud phase and AIRS phase decisions that are collocated with groups of CCL FOVs for two separate granules in summer and winter. 


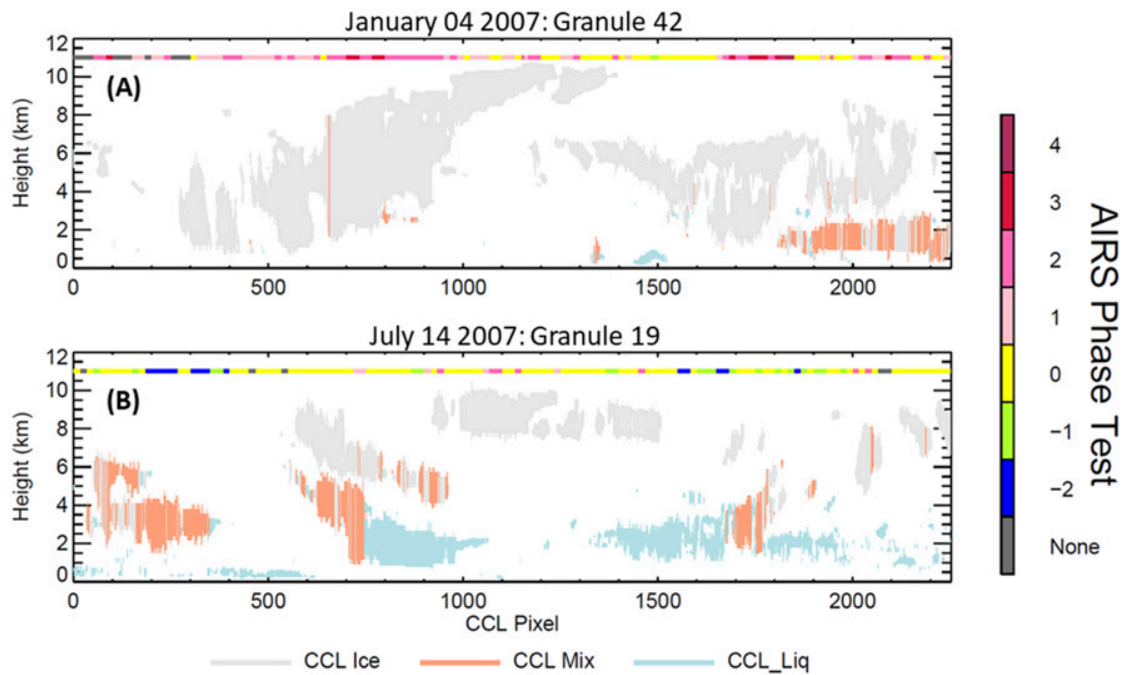

FIG. 1. Vertical profile of individual cloud layers detected by CCL along an AIRS-CCL collocated ground track (a) on 4 Jan 2007 from $76.26^{\circ} \mathrm{N}, 106.51^{\circ} \mathrm{E}$ to $76.69^{\circ} \mathrm{N}, 1.6^{\circ} \mathrm{W}$ and (b) on $14 \mathrm{Jul} 2007$ from $80.8^{\circ} \mathrm{N}, 60.62^{\circ} \mathrm{E}$ to $63.85^{\circ} \mathrm{N}, 12.1^{\circ} \mathrm{E}$. The horizontal colored bar at $11 \mathrm{~km}$ represents AIRS phase decisions for AIRS FOVs overlapping with groups of CCL FOVs.

For the MODIS case study described in section 4, 1-km MODIS pixels are collocated within each AIRS FOV where CCL is also collocated in the AIRS FOV during the month of July 2009 for daytime only. The collocation is based on the method developed by Schreier et al. (2010). There are 200 MODIS $1-\mathrm{km}$ pixels for a 13.5-km nadir AIRS FOV.

\section{f. Cloud amount and layering considerations for AIRS cloud phase evaluations}

\section{1) Selection of CCL ClOUd LAyering in AIRS FOVS}

Cho et al. (2009) and JN14 both reported that, for MODIS and AIRS, respectively, a higher cloud phase classification skill is achieved for single-layer cloud systems. However, multilayer cloud systems occur frequently on a global scale and should not be ignored. Since this study focuses on the Arctic, it is important to first evaluate the frequency of occurrence of different cloud-layer amounts in the Arctic. Figure 2 shows the relative frequency of occurrence of the cloud-layer amount using groups of CCL pixels where $\geq 90 \%$ of the collocated CCL FOVs within the AIRS FOVs are either all covered by single-layer clouds or all covered by two-layer clouds over open ocean and sea ice. These will be referred to as single-layer-overcast and twolayer-overcast CCL FOV groups. Regardless of the surface type, single-layer-overcast systems are the most prevalent $(75 \%-80 \%)$ while two-layer-overcast systems are the second most common $(15 \%-20 \%)$. A similar conclusion is drawn using cloud-layer numbers from individual CCL FOVs (not shown). CCL FOV groups with more than two layers are scarce in the collocated observations. It is possible that some single-layer clouds detected by CCL may have underlying cloud layers, but these lower layers may not be detected by CCL due to full attenuation of the CALIPSO lidar, which occurs for the cloud layer with a visible optical depth greater than $\sim 3$. Given the cloud layering statistics described here,

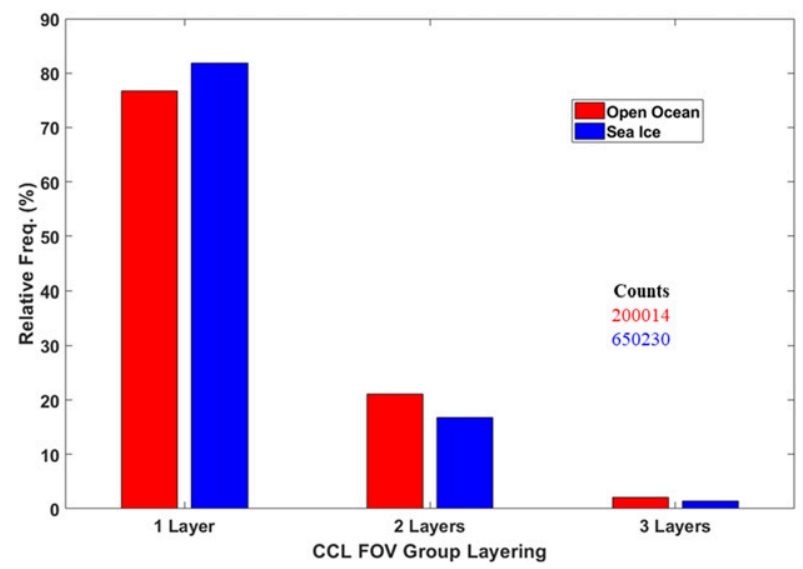

FIG. 2. Relative frequency of occurrence of overcast CCL FOV groups with a given layering number for sea ice and open ocean. For the layers to be counted, $\geq 90 \%$ of CCL FOVs must detect cloud and must all have the same number of layers. The normalization is with respect to all FOV groups over each respective surface type. The counts represent the number of CCL FOV groups included for each surface type. 

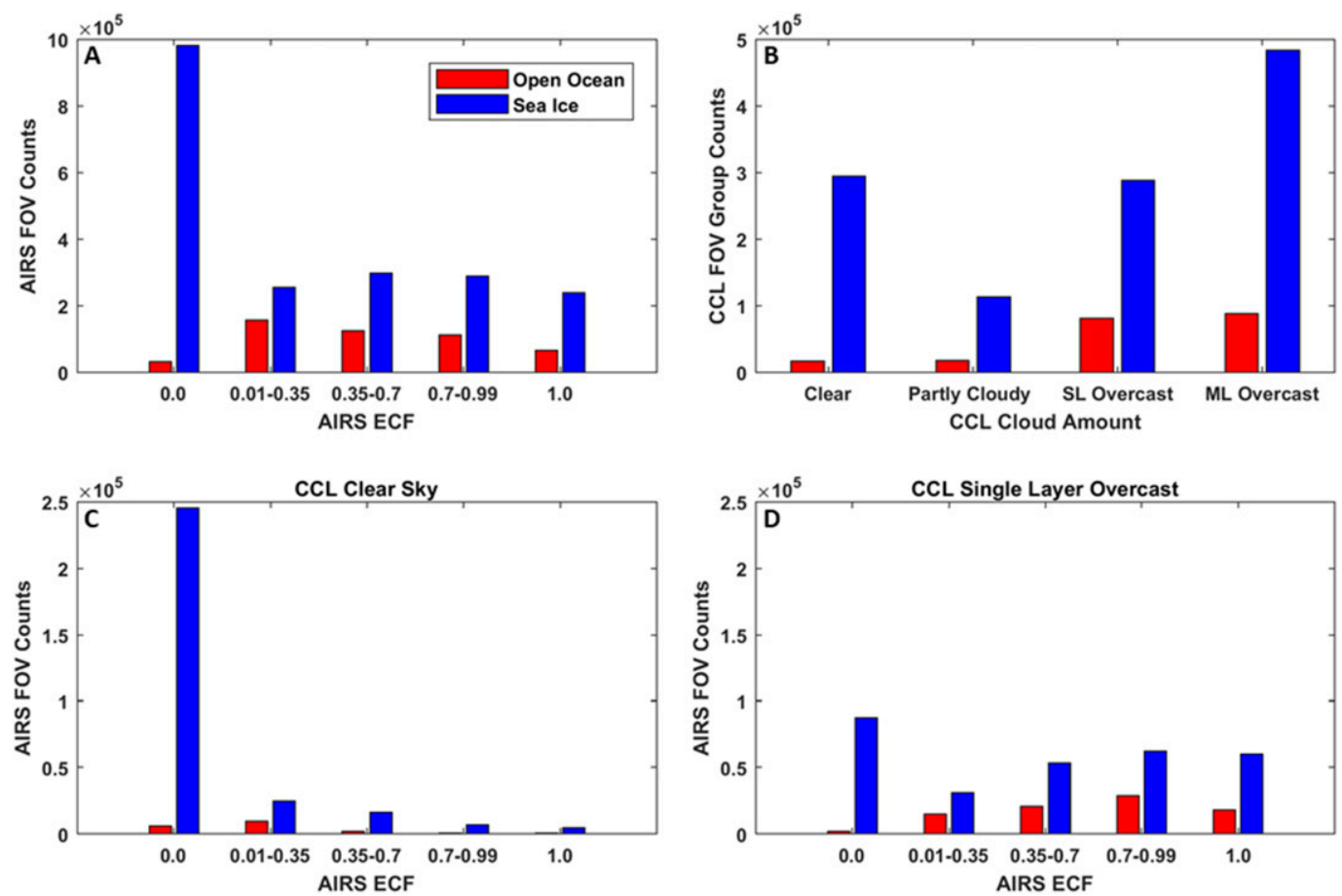

FIG. 3. (a) Counts of AIRS FOVs over sea ice and open ocean separated on the basis of AIRS ECF. (b) Counts of CCL FOV groups collocated within AIRS FOVs over sea ice and open ocean for four CCL cloud amount categories. CCL clear sky is defined as $\geq 90 \%$ of the CCL FOVs with no cloud layer. CCL partly cloudy is defined as between $10 \%$ and $90 \%$ of CCL FOVs with clouds detected. Single-layer (SL) overcast is for $\geq 90 \%$ of CCL FOVs detected as single-layer clouds, and multilayer (ML) overcast is for $\geq 90 \%$ of CCL FOVs with clouds detected with at least one FOV having more than one layer of cloud. (c) As in (a), but only AIRS FOVs corresponding to CCL FOV clear-sky groups are included. (d) As in (c), but for the CCL FOV group being SL overcast.

we use single-layer and two-layer CCL FOV groupings in the AIRS phase evaluation.

\section{2) SELECTION OF CCL CLOUd COVER IN AIRS FOVS}

In addition to assessing the consistency of cloud phase classifications between AIRS and CCL, it is also important to assess the qualitative cloud detection consistency of the AIRS and CCL products. Such an evaluation will determine if AIRS cloud detection is consistent with the single-layer-overcast CCL groups, which are the main CCL groups used in the AIRS cloud phase evaluation. Figure 3 shows the occurrence of AIRS FOVs within five ECF intervals compared with CCL FOV groups with different cloud amounts. Also, the distribution of AIRS ECF occurrence within two different CCL cloud amount categories is explored. It is important to note that AIRS ECF and CCL cloud amount are physically different and the comparison here is qualitative and relative.

Four single-layer CCL cloud amount categories are created for CCL FOV groups within AIRS FOVs: 1) CCL clear-sky when $\geq 90 \%$ of the CCL FOVs indicate no cloud layer, 2) CCL partly cloudy conditions when between $10 \%$ and $90 \%$ of CCL FOVs are covered by single-layer or multilayer clouds, 3 ) the aforementioned single-layer-overcast grouping, and 4) multilayer-overcast conditions that include all cases in which $\geq 90 \%$ of CCL FOVs detect clouds with at least one FOV having more than one cloud layer.

Figures $3 \mathrm{a}$ and $3 \mathrm{~b}$ show a comparison between counts of AIRS FOVs in five ECF intervals and CCL groups in four CCL cloud amount categories over sea ice and open ocean. Over open ocean, most AIRS FOVs and CCL FOV groups indicate cloud presence, which is consistent with the annual peak of cloud amount occurring in the Arctic summer and autumn when open ocean represents a large fraction of surface area. Over sea ice, both AIRS and CCL show that the fractions of cloudy and clear-sky AIRS FOVs, as detected by the AIRS and CCL algorithms, respectively, are more comparable. This is consistent with the fact the cloud cover minimum in the Arctic occurs in the winter and spring when there is more sea ice coverage. CCL partly cloudy groups occur less often than overcast and clear-sky CCL groups, which is consistent with the overall high cloud cover observed in the Arctic throughout the year. 

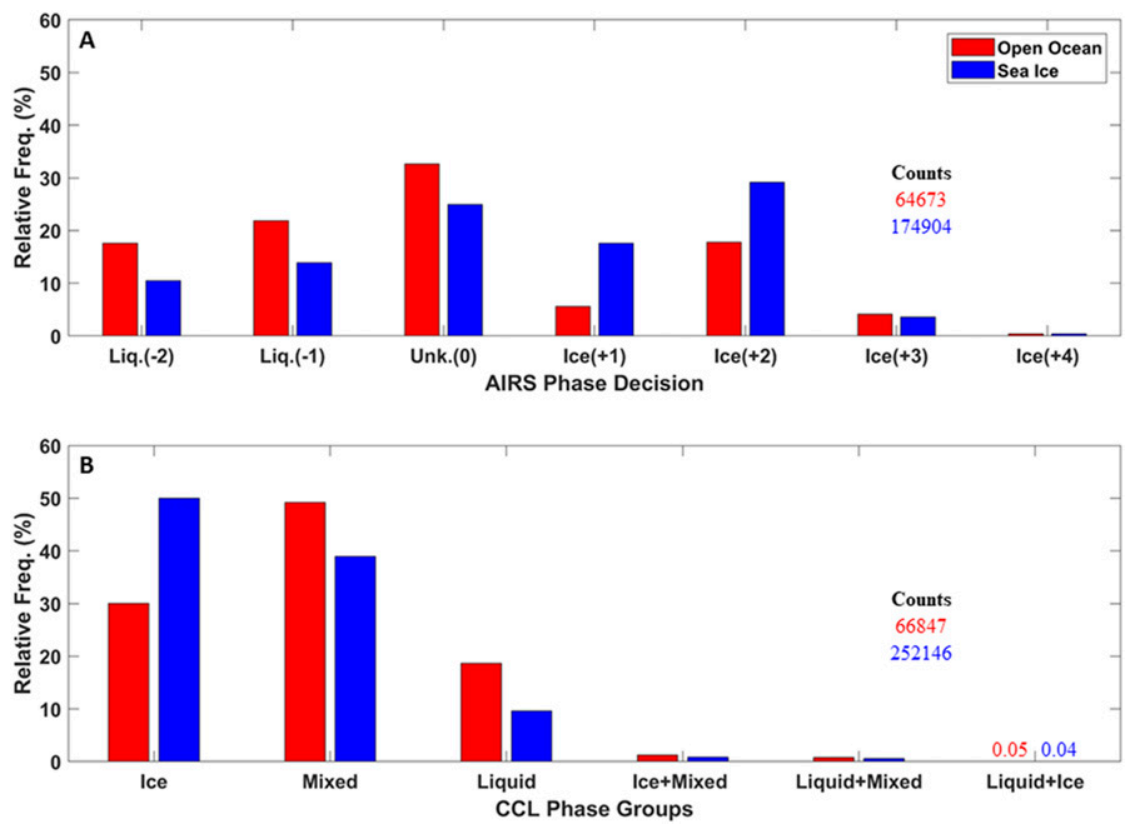

FIG. 4. (a) Frequency of occurrence of AIRS phase decisions over open ocean and sea ice for only those AIRS FOVs that coincide with a single-layer-overcast homogenous- or heterogeneousphase CCL FOV group. The counts represent the number of AIR FOVs included in the analysis for each surface type, and the normalization is with respect to these counts. (b) Frequency of occurrence of homogenous- and heterogeneous-phase groupings of singlelayer-overcast CCL FOV groups collocated with AIRS. Ice, liquid, and mixed-phase groups represent CCL homogenous-phase groups, and the ice + liquid, ice + mixed, and liquid + mixed are for the CCL heterogeneous-phase groups. The counts represent the number of CCL FOV groups included in the analysis for each surface type. The normalization is with respect to these counts. Numerical percentages for each surface type replace bars when the bars are not visible.

Figures $3 \mathrm{c}$ and $3 \mathrm{~d}$ show AIRS ECF occurrence within the CCL clear-sky and CCL single-layer-overcast groups, respectively. Most AIRS FOVs in which AIRS detects cloud $(\mathrm{ECF}>0)$ fall into the CCL overcast and partly cloudy categories regardless of surface type. However, over sea ice, about one-quarter of clear-sky AIRS FOVs $(\mathrm{ECF}=0)$ fall into the CCL clear-sky category and the rest fall into the CCL overcast and partly cloudy categories, and this suggests that AIRS can misidentify cloudy scenes as clear over sea ice from a lack of thermal contrast between the surface and cloud top. Overall, the single-layer-overcast CCL FOV groupings are associated with AIRS FOVs that will have a cloud phase assigned (i.e., when ECF > 0.01), which further justifies the use of such overcast CCL groupings for AIRS cloud phase evaluation.

\section{Results and discussion}

\section{a. Overview of Arctic cloud phase occurrence using collocated AIRS and CCL FOVS}

For an initial comparison between AIRS and CCL Arctic cloud phase, the occurrence of AIRS and CCL cloud phase over sea ice and open ocean is examined using the collocated AIRS-CCL FOV dataset. Different homogenous- and heterogeneous-phase categories are defined using collocated single-layer-overcast CCL FOVs within the AIRS FOV as follows: homogenous ice-, liquid-, and mixed-phase CCL groups when $\geq 90 \%$ of the CCL FOV groups have that phase, respectively, and the heterogeneous-phase groups (ice + liquid, liquid + mixed, ice + mixed) when each phase in any two-phase mixture makes up $45 \%-55 \%$ of the CCL FOV group. The heterogeneous groups are defined similarly to the JN14 mixed-phase category. As was mentioned in section 2a, the AIRS cloud phase algorithm employs 4 ice tests and 2 liquid tests. The results of all tests are summed and the classification is ice if the value is positive (from +1 to +4 ), unknown if 0 , and liquid if negative $(-1$ and -2$)$.

Figure 4 shows the relative frequency of occurrence of cloud phase over sea ice and open ocean for the homogenous and heterogeneous-phase CCL groups (Fig. 4b) and by the AIRS phase algorithm in which the AIRS FOVs are subsampled based on collocation with these CCL groups (Fig. 4a). Since homogenous-phase CCL 

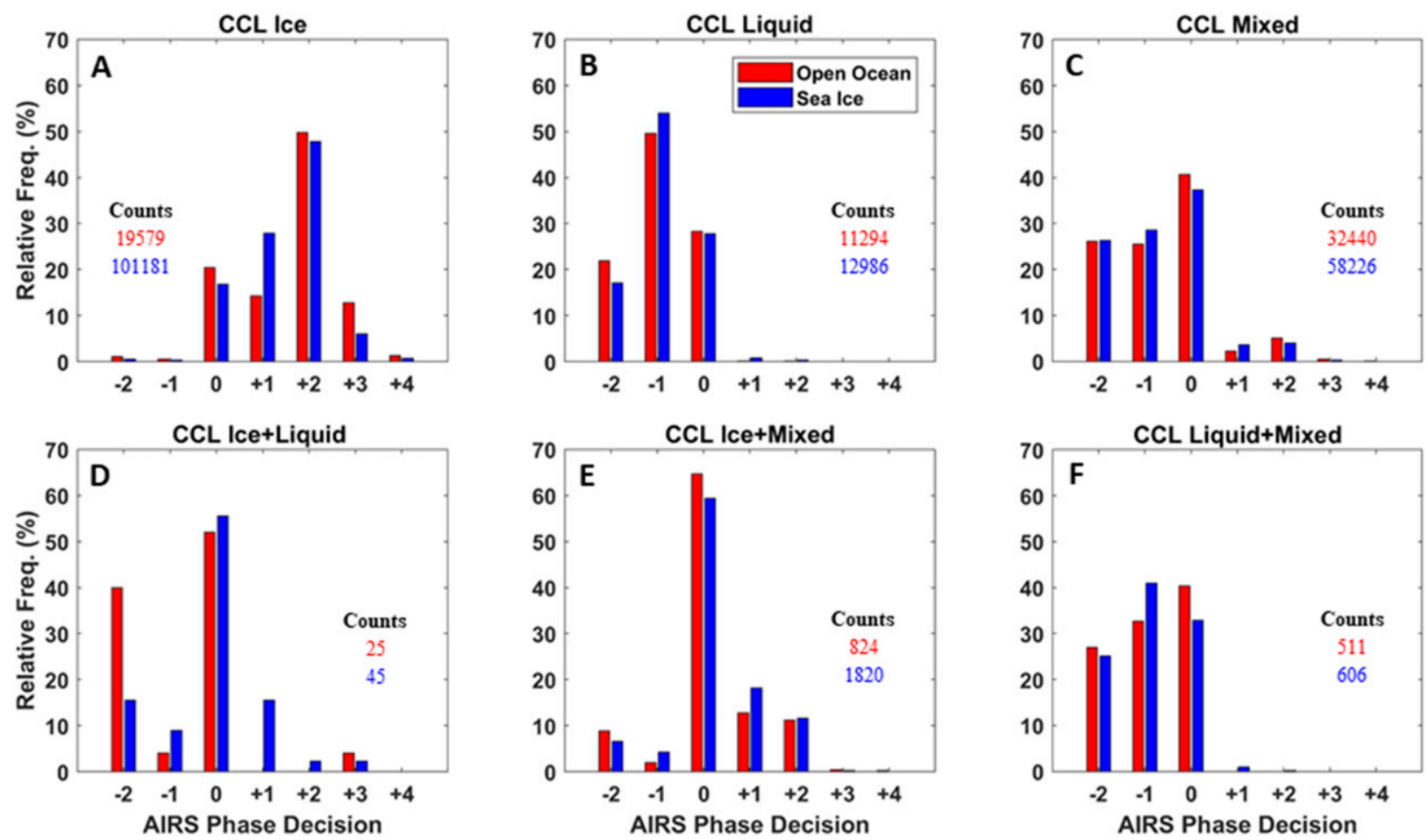

FIG. 5. Frequencies of occurrence of AIRS phase classifications for AIRS FOVs corresponding to single-layer-overcast groups of CCL FOVs with a (a)-(c) homogenous- or (d)-(f) heterogeneous-phase type. The counts represent the number of AIRS FOVs included in the analysis for each surface type. Normalization is with respect to these counts.

occurrence is dominant, only these groups will be discussed here. CCL mixed phase is the most dominant cloud type over open ocean, where its occurrence is nearly $50 \%$. Over sea ice, the occurrence of CCL mixed phase is $\sim 40 \%$. Over open ocean, $30 \%$ of CCL groups are ice phase, where ice-phase occurrence for AIRS is $27.9 \%$. Over sea ice, the ice-phase occurrence is $50 \%$ for CCL and $50.7 \%$ for AIRS ice phase. For CCL liquid phase over open ocean, the occurrence is $20 \%$, where for AIRS liquid phase it is $39.5 \%$. Over sea ice, the CCL liquid-phase occurrence is $10 \%$ and $23.3 \%$ for AIRS liquid phase.

When considering AIRS FOVs that are collocated with homogenous-phase CCL groups, the frequency of occurrence of AIRS and CCL ice phase agree within $2.5 \%$, while AIRS tends to overestimate the occurrence of CCL liquid phase by as much as $20 \%$. Overall, $27 \%$ of the AIRS Arctic cloud phase classifications associated with the homogenous and heterogeneous-phase CCL groups are unknown phase, and $42 \%$ are unknown phase when considering all AIRS Arctic FOVs with a cloud phase assigned. This prompts a thorough examination of the conditions associated with these unknown-phase classifications as well as other AIRS phase classifications, which will be discussed in the following section.

\section{b. AIRS Arctic cloud phase evaluations}

\section{1) Single-LAYER SYSTEMS}

To evaluate AIRS Arctic cloud phase classifications against single-layer-overcast CCL groups, we first assess AIRS classification tendency for various CCL phase groupings and subsequently evaluate AIRS phase classification skill for the Arctic.

Figure 5 shows the frequency of occurrence of AIRS phase classifications partitioned based on AIRS cloud phase test summations for single-layer-overcast homogenous and heterogeneous-phase CCL groupings. Overall, the percentage of homogenous-phase CCL groups classified as a particular AIRS phase is not dependent on surface type. AIRS classifies $78 \%$ of homogeneous CCL ice-phase groups over open ocean (82.4\% over sea ice) as ice phase and the rest as unknown phase (Fig. 5a). This high rate of agreement between AIRS and CCL ice-phase scenes is consistent with AIRS being more sensitive to ice phase than liquid phase (Kahn et al. 2014), and JN14 reported a similar result for AIRS comparisons with CALIPSO on the global scale. For homogenous CCL liquid-phase groups over open ocean, AIRS classifies $71.5 \%$ as liquid phase 
(71\% over sea ice) and the rest as unknown phase (Fig. 5b). JN14 found that on a global scale, $\sim 40 \%$ of homogenous CALIPSO liquid-phase groups were classified as liquid phase by AIRS, as compared with $\sim 70 \%$ in this study. It is possible that a higher fraction of liquid clouds can be classified correctly in the Arctic relative to lower latitudes because of the drier Arctic atmosphere, especially since liquid clouds tend to occur lower in the atmosphere.

More interesting are the homogenous CCL mixedphase groups (Fig. 5c). Over open ocean, AIRS classifies $52 \%$ of these groups as liquid, $41 \%$ as unknown, and $8 \%$ as ice phase. For sea ice, it is $55 \%, 38 \%$, and $8 \%$ for AIRS liquid, unknown, and ice phase, respectively. (Note that rounding leads to $101 \%$ in both cases.) This result can be contrasted against the results of JN14 where the majority of CALIPSO mixed-phase groups were classified as unknown phase by AIRS. The tendency for AIRS to classify the homogenous mixedphase CCL groups as liquid phase could be related to the structure of Arctic mixed-phase clouds, as such clouds tend to have a thin liquid layer at the cloud top (e.g., Morrison et al. 2012).

The AIRS phase classifications of heterogeneousmixed-phase CCL groups appear to differ from that of the homogenous-mixed-phase CCL groups, but it is also important to note that there are fewer samples in the heterogeneous-phase cases. For the heterogeneous ice + liquid and ice + mixed CCL groups (Figs. 5d,e), most of the AIRS phase classifications are unknown, which is similar to the results of JN14. Note that the JN14 CALIPSO mixed-phase groups are like the CCL heterogeneous-mixed-phase groups. AIRS classified relatively more of the liquid + mixed CCL phase groups as liquid phase when compared with the homogenousmixed-phase CCL groups (Fig. 5f), which may simply be a result of more liquid cloud being present in the AIRS FOV. The results here suggest that AIRS phase classifications could be dependent on the spatial scale of cloud phase mixing.

Table 1 shows percentage composition of all AIRS FOVS classified as AIRS liquid, ice, or unknown phase in terms of the homogenous-phase CCL groups. This represents an assessment of AIRS classification skill in the Arctic. Over open ocean, $86 \%$ of AIRS FOVs classified as ice phase by AIRS were classified as ice phase by CCL, where over sea ice the ice-phase classification skill is $95 \%$. The majority of AIRS liquid-phase classifications correspond to CCL mixed phase over both surface types. For open ocean, the percentage of AIRS FOVs classified as liquid phase by AIRS that are classified as mixed phase by CCL is $67 \%$ and over sea ice the value is $76 \%$. AIRS liquid-phase classification skill
TABLE 1. AIRS phase classification skill matrix: percentage composition of all AIRS FOVs with a given phase classification in terms of CCL phase using homogenous single-layer-overcast CCL FOV groups over open ocean. The values in parentheses are for sea ice.

\begin{tabular}{lccc}
\hline \hline \multicolumn{1}{c}{ Phase class } & CCL ice & CCL liquid & CCL mixed \\
\hline AIRS ice & $85.76(94.64)$ & $0.13(0.17)$ & $14.11(5.18)$ \\
AIRS liquid & $1.2(1.88)$ & $32.13(21.98)$ & $66.65(76.14)$ \\
AIRS unknown & $19.62(40.1)$ & $15.69(8.53)$ & $64.68(51.37)$ \\
\hline
\end{tabular}

differs over the surface types, where it is $32 \%$ over open ocean and $22 \%$ over sea ice. It is possible that the $\sim 10 \%$ difference in AIRS liquid and ice-phase classification skill over the surface types is related to differences in the surface emissivity of the surfaces for cases in which the cloud is not entirely opaque.

AIRS unknown-phase classifications are mainly composed of CCL ice-phase and CCL mixed phase, but the relative contribution of these CCL phase groups is different over each surface type. Over sea ice, $40 \%$ of AIRS FOVs classified as AIRS unknown phase are classified as ice phase by CCL and $51 \%$ are classified as mixed phase by CCL. Over open ocean, it is $19 \%$ and $65 \%$ for CCL ice phase and mixed phase, respectively.

Overall, mixed-phase clouds appear to contribute the most to AIRS unknown- and liquid-phase classifications in the Arctic. As discussed previously, the liquid-topped structure of mixed-phase clouds could contribute to AIRS liquid-phase classifications, but if the upper region of the mixed-phase cloud is more homogenously mixed in terms of ice and liquid particles, this could lead to AIRS unknown-phase classifications.

\section{2) TWO-LAYER SYSTEMS}

Two-layer-overcast CCL groups occur $15 \%-20 \%$ of the time in the Arctic as compared with all overcast CCL groups in which $\geq 90 \%$ CCL FOVs have the same layer number. Figure 6 a shows the frequency of occurrence of two-layer phase combinations in which each layer are overcast CCL groups with homogenous phase. The most common two-layer phase systems are ice phase over liquid phase and ice phase over mixed phase. Such twolayer systems typically consist of ice clouds above $4 \mathrm{~km}$ and lower-level liquid and mixed-phase clouds, which is illustrated in Fig. 1.

Figures $6 \mathrm{~b}$ and $6 \mathrm{c}$ show the relative frequency of occurrence of AIRS phase decisions for the ice-phaseover-liquid-phase and ice-phase-over-mixed-phase groups, respectively. For both groups, regardless of surface type, most AIRS classifications are unknown phase, but a nonnegligible amount of AIRS FOVs are classified as liquid and ice phase for both two-layer groups. 

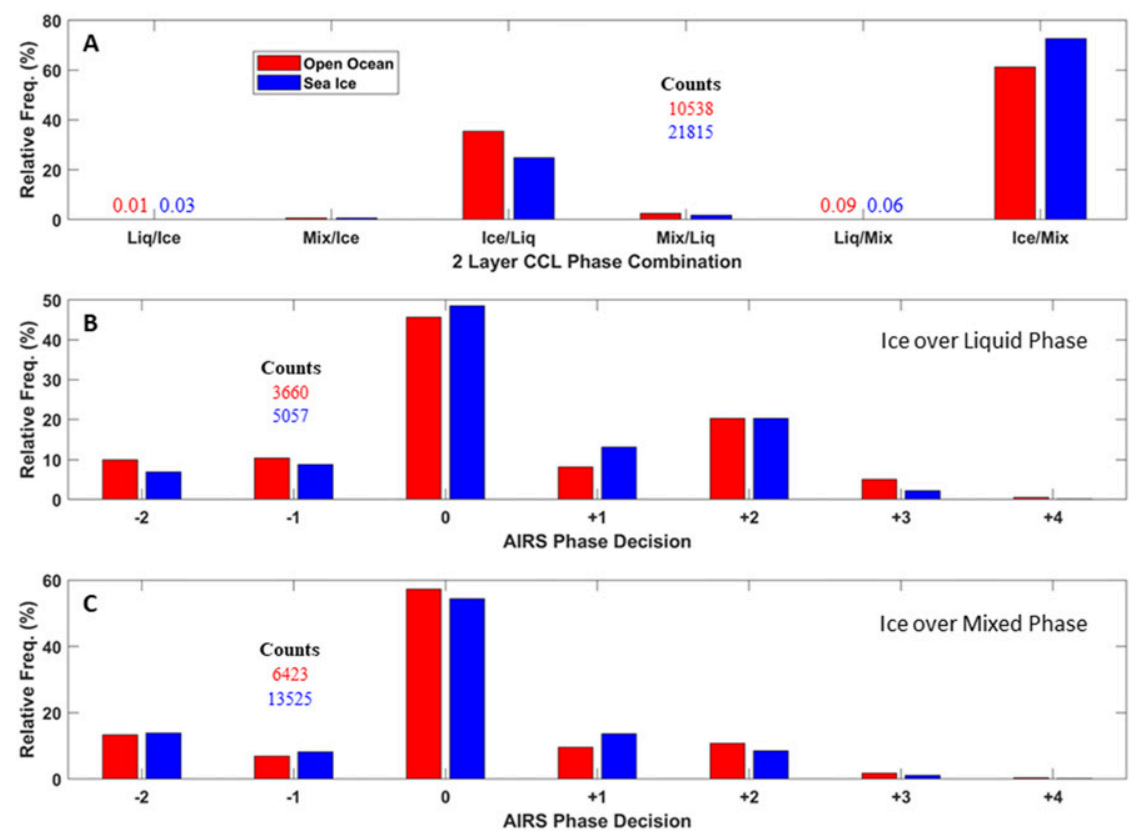

FIG. 6. (a) Frequency of occurrence of two-layer-overcast CCL phase groups over open ocean and sea ice. The counts represent the number of CCL groups included in the analysis for each surface type. Normalization is with respect to these counts. (b) Frequency of occurrence AIRS phase decisions for two-layer-overcast CCL ice phase over liquid phase for open ocean and sea ice. The counts represent the number of AIRS FOVs included in the analysis for each surface type. (c) As in (b), but for CCL ice phase over mixed phase. Numerical percentages for each surface type replace bars when the bars are not visible.

For the cases in which the upper-layer ice cloud is optically thick in the IR, it is expected that most of those cases will be classified as ice phase by AIRS given the high ice-phase classification skill. When the upper layer is not completely opaque in the IR, the underlying mixed-phase or liquid-phase layer could be contributing to the AIRS liquid- and unknownphase classifications.

\section{3) AIRS PHASE EVALUATION IN THE VERTICAL DIMENSION}

Figure 7 shows the frequency of occurrence of CCL cloud phase from individual CCL cloud layers that are binned based on the overlying AIRS phase decision and surface type. The cloud layers are shown for 39 altitude bins from 0 to $10 \mathrm{~km}$. All CCL FOVs that have successful cloud phase detections are used, including those with vertical layer numbers larger than 1 . This provides another perspective for evaluating AIRS cloud phase classifications in comparison with the overcast CCL FOV grouping methods previously shown.

AIRS FOVs classified as ice phase by AIRS tend to correspond to the presence of CCL ice cloud layers above $4 \mathrm{~km}$ (Figs. 7c,f), which is consistent with the high ice-phase classification skill of AIRS (Table 1).
There are also CCL liquid- and mixed-phase layers present $\sim 40 \%$ of the time over open ocean and $\sim 20 \%$ of the time over sea ice (Figs. 7c,f), which are mostly excluded when selecting only overcast groups of CCL FOVs. The vertical distribution of CCL phase within AIRS FOVs classified as liquid phase by AIRS over open ocean (Fig. 7b) shows very little CCL ice-phase layers $(<5 \%)$ and a comparable mixture of liquid(from 0 to $4 \mathrm{~km}$ ) and mixed-phase (from 0 to $6 \mathrm{~km}$ ) CCL layers. A similar vertical distribution of CCL phase within AIRS FOVS classified as liquid by AIRS can also be seen over sea ice (Fig. 7e), but in this case mixed-phase clouds are more dominant relative to open ocean. This is consistent with AIRS liquid classifications having most contributions from CCL liquid- and mixedphase groupings, and more AIRS liquid phase being classified as mixed phase by CCL over sea ice (Table 1 ). For the AIRS unknown-phase category (Figs. 7a,d), the occurrence of CCL ice-, liquid-, and mixed-phase cloud layers are more comparable from 0 to $10 \mathrm{~km}$, with the largest contributions coming from CCL mixed phase over both surface types, which is a feature also seen in Table 1. The larger rate of misclassification of CCL ice-phase groups as unknown by AIRS over sea ice (see Table 1) is also seen here in the vertical cloud information. 

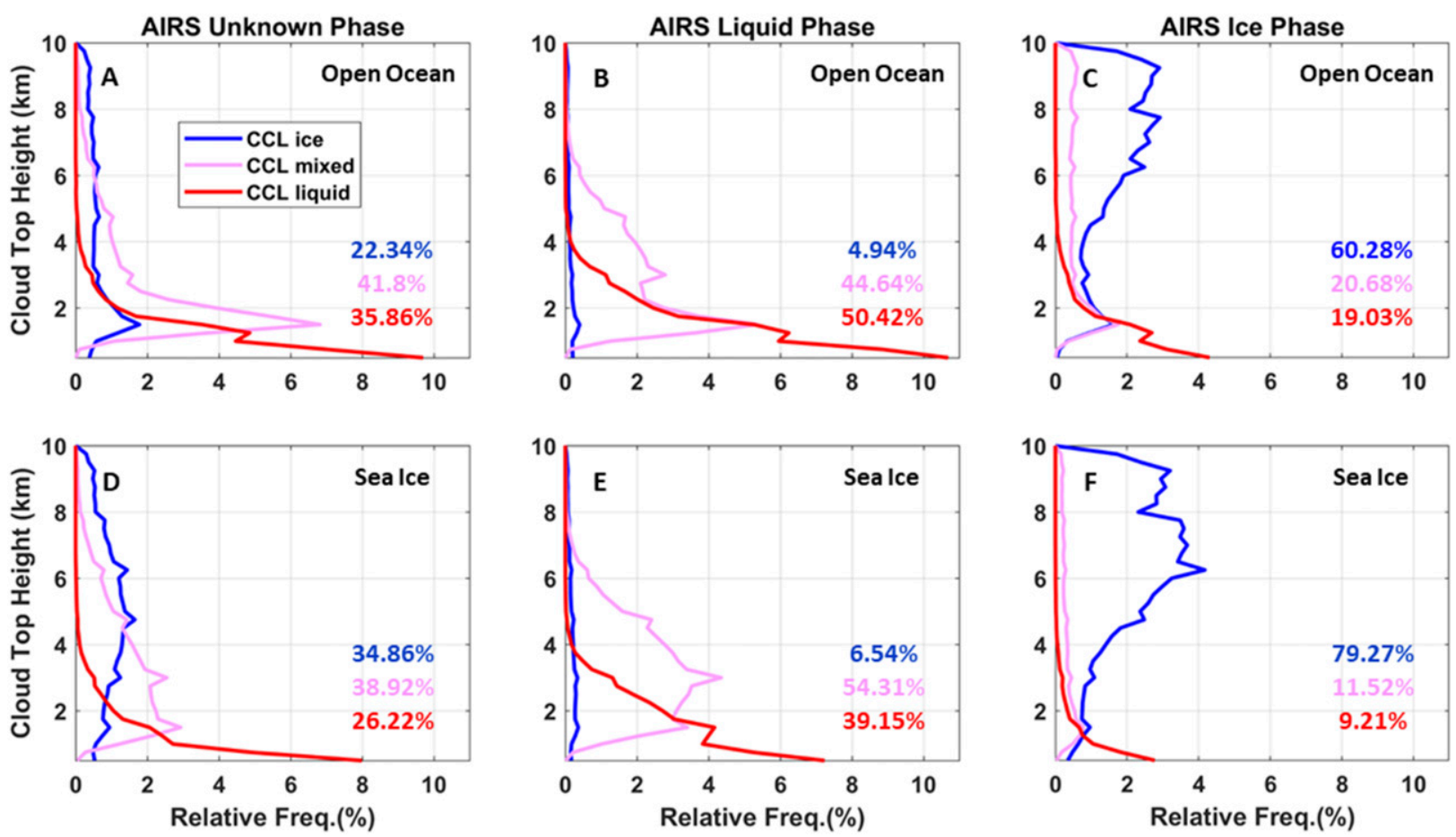

FIG. 7. Frequency of occurrence of individual mixed-phase, ice, or liquid CCL layers that are binned on the basis of the overlying AIRS phase classification over (a)-(c) open ocean and (d)-(f) sea ice. Each individual CCL layer within a single CCL FOV is counted here separately, and there are 39 altitude bins from 0 to $10 \mathrm{~km}$. The percentages represent the vertical sums of each phase type frequency for each panel. The normalization is with respect to the total number of cloud layers detected by CCL that occur within an AIRS phase category over each respective surface type.

\section{c. Climatological perspectives of AIRS and CCL Arctic cloud phase comparisons}

In this section, the evaluation of AIRS cloud phase retrievals in the Arctic is extended to a climatological context in which AIRS and CCL cloud phase from the collocated AIRS-CCL dataset is compared seasonally, zonally, and by compositing by TCWV and the temperature difference between 1000 and $300 \mathrm{hPa}\left(\Delta T_{1000-300}\right)$. The composite with respect to TCWV and $\Delta T_{1000-300}$ is used to examine the cloud phase classification with respect to large-scale dynamic and thermodynamic factors, in addition to the zonal and seasonal characterization of the cloud phases as identified by the AIRS and CCL algorithms. Here, all collocated AIRS and CCL FOVs with a cloud phase assigned are used. For the TCWV and $\Delta T_{1000-300}$ composites, AIRS and CCL FOVs were matched with the nearest hourly $0.25^{\circ} \times$ $0.25^{\circ}$ ERA5 grid.

\section{1) SEAsonal AND ZONAL COMPARISONS}

Figure 8 shows the relative frequency of occurrence of AIRS and CCL cloud phase using all CCL cloud layers for $5^{\circ}$ zonal bands during all four seasons (Figs. 8a-d) and the Arctic-wide vertical frequency of occurrence of CCL cloud phase using all layers during each season (Figs. 8e-h). All surface types are used here.

For all seasons and latitudes, the relative occurrence of liquid phase is similar for AIRS and CCL (Figs. 8a-d), where the differences tend to be within $10 \%$. Furthermore, AIRS underestimates ice-phase relative occurrence when compared with CCL during all seasons, where the difference in relative occurrence between AIRS and CCL ice phase ranges from $\sim 10 \%$ to $30 \%$ depending on the latitude and season. It is important to note here that AIRS ice and liquid-phase occurrence is relative to AIRS unknown phase while CCL ice and liquid-phase occurrence is relative to CCL mixed phase. Given that most AIRS unknown-phase classifications correspond to CCL ice and mixed-phase groups, it is unsurprising that the liquid-phase relative occurrence agrees more between the instruments. When the AIRS and CCL cloud phases are compared seasonally and zonally using the single-layer-overcast and homogenous-phase CCL groups only (not shown), the ice-phase occurrence between the instruments agrees more than for liquid phase, which is consistent with AIRS phase classification skill shown in Table 1.

Although there are differences in the magnitude of relative occurrence of cloud phase from AIRS and CCL, 

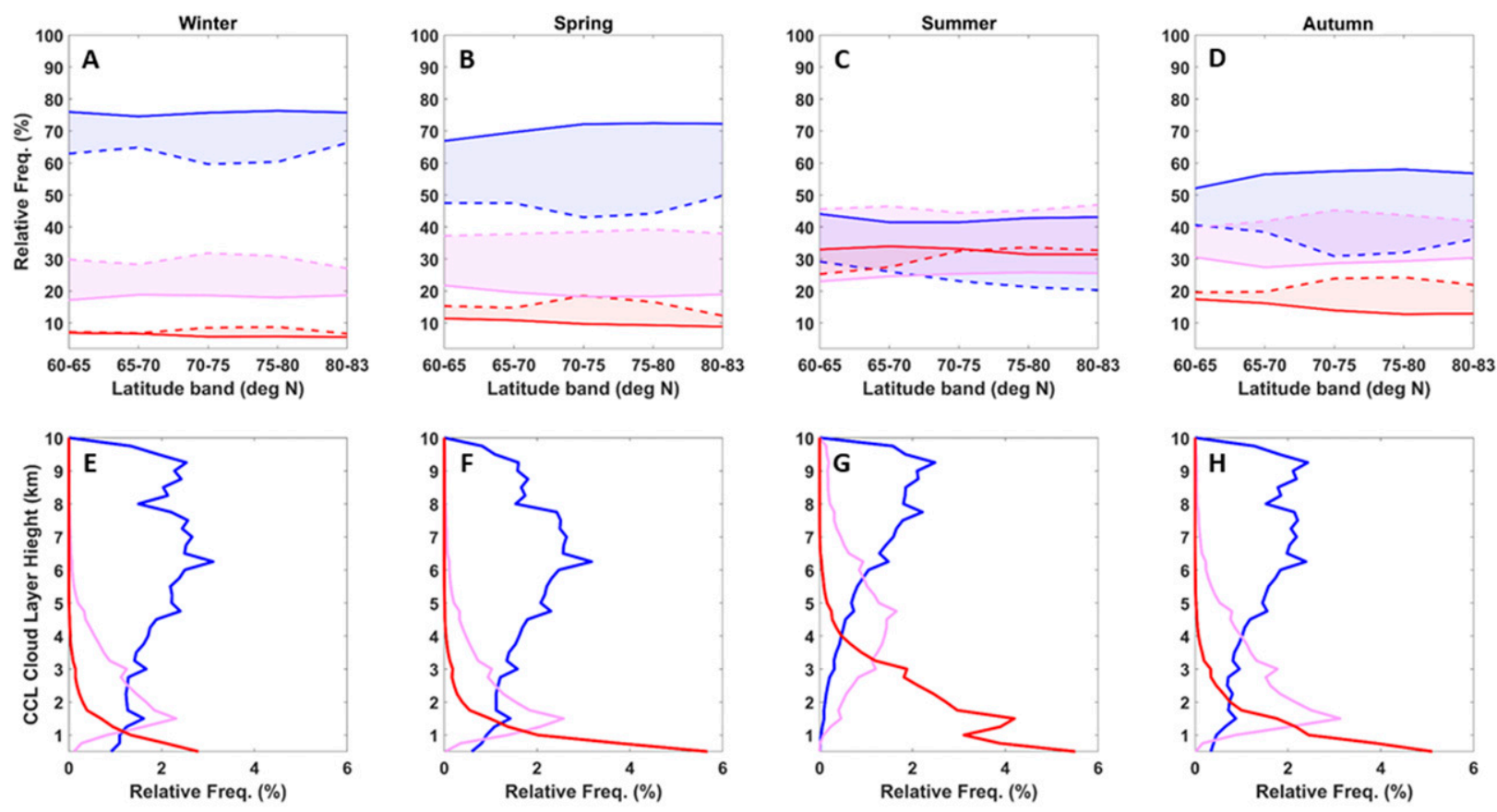

FIG. 8. Frequency of occurrence of CCL phase using all CCL detected cloud layers (solid lines) and AIRS cloud phase (dashed lines) for $5^{\circ}$ latitudinal bands (except for $80^{\circ}-83^{\circ} \mathrm{N}$ ) for (a) winter, (b) spring, (c) summer, and (d) autumn. All surface types are included. The frequencies are relative to the total FOV count in each latitudinal band for each instrument for each season. Also shown are frequency of occurrence of individual mixed-phase, ice, or liquid CCL layers over all surface types during (e) winter, (f) spring, (g) summer, and (h) autumn. Each individual CCL layer within a single CCL FOV is counted here separately, and there are 39 altitude bins from 0 to $10 \mathrm{~km}$. The normalization is with respect to the total number of cloud layers detected by CCL during a season.

the relative occurrence of cloud phase from each instrument follow a similar seasonal cycle (Figs. 8a-d), where ice and liquid phase peak in the winter and summer, respectively. Overall, AIRS unknown-phase classifications tend to follow the seasonal change in CCL mixed phase, as most AIRS unknown-phase classifications correspond to CCL mixed phase (Table 1). However, a significant fraction of AIRS unknown-phase classifications also correspond to CCL ice phase as well, and therefore the opposing seasonal cycles of CCL ice and mixed/liquid phase can act to dampen changes in AIRS unknown-phase occurrence across seasons.

The seasonal cycle of the vertical relative frequency of occurrence of CCL phase (Figs. 8e-h) shows agreement with the zonal patterns. In winter and spring, most clouds are ice phase and occur throughout the atmosphere and peak at $\sim 6 \mathrm{~km}$. In summer and autumn, the overall occurrence of liquid phase increases, mainly below $4 \mathrm{~km}$. CCL mixed-phase occurrence peaks in autumn at $\sim 2 \mathrm{~km}$.

\section{2) TOTAL COLUMN WATER VAPOR COMPOSITE}

Figure 9 shows the relative frequency of occurrence of AIRS cloud phase and CCL cloud phase using all
CCL detected cloud layers over sea ice and open ocean for TCWV intervals spanning $1-20 \mathrm{~kg} \mathrm{~m}^{-2}$. Over open ocean, most cloudy AIRS and CCL FOVs are associated with the TCWV range of $4-12 \mathrm{~kg} \mathrm{~m}^{-2}$ (Figs. 9a,c). This can be contrasted with the sea ice scenes, where the regime shifts to $1-4 \mathrm{~kg} \mathrm{~m}^{-2}$ (Figs. 9b,d). This is consistent with sea ice being associated with colder and drier regions and the largely reduced local evaporation.

Over both surface types, CCL liquid-phase occurrence relative to the other CCL phases has a similar magnitude and relationship with TCWV, where it increases from $5 \%-10 \%$ to $30 \%-35 \%$ as $\mathrm{TCWV}$ is increased (Figs. 9a,b). The presence of more liquid clouds relative to the other phases at higher TCWV values is likely physical. Over open ocean, mixed-phase cloud relative occurrence is largest at $40 \%$ in the driest TCWV regimes and decreases to $30 \%$ as TCWV is increased. This relationship between CCL mixed phase and TCWV is different over sea ice, where mixed-phase cloud relative occurrence is largest in the wettest TCWV regime. Such differences highlight the complex relationship between Arctic mixed-phase clouds and humidity. For CCL ice-phase occurrence, the relationship between 

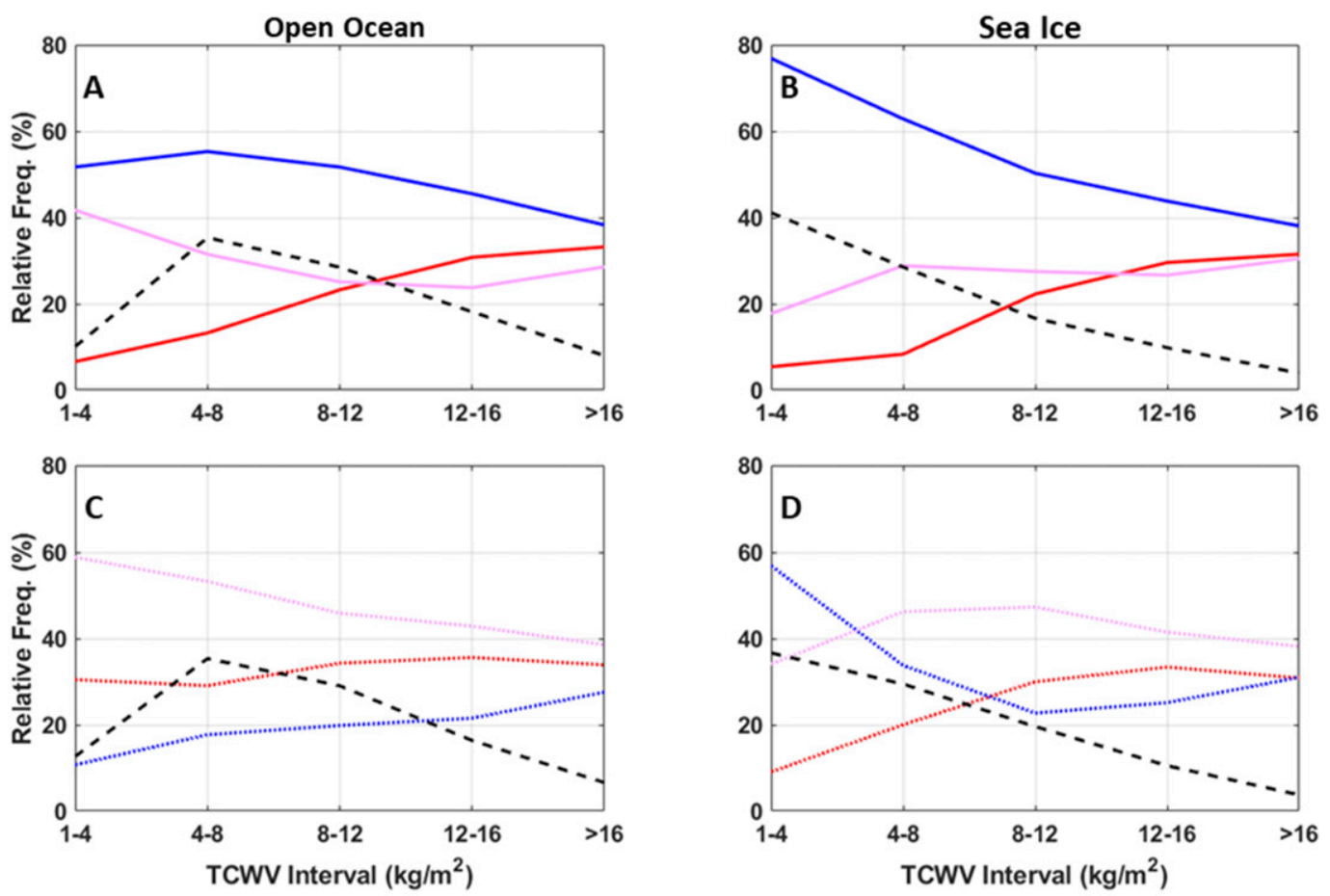

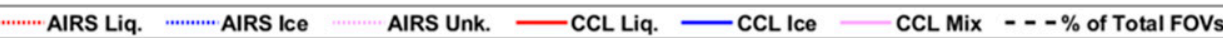

FIG. 9. (a) Frequency of occurrence of CCL cloud phase using all CCL detected cloud layers over open ocean for intervals of TCWV. The dotted black line represents the percentage of cloudy CCL FOVs that fall within a TCWV interval. Normalization is with respect to all CCL FOVs with a cloud phase assigned in a given TCWV interval. (b) As in (a), but for sea ice. (c) Frequency of occurrence of AIRS cloud phase over open ocean for intervals of TCWV. The dotted black line represents the percentage of cloudy AIRS FOVs that fall within a TCWV interval. Normalization is with respect to all AIRS FOVs with a cloud phase assigned in a given TCWV interval. (d) As in (c), but for sea ice.

TCWV and ice-phase cloud relative occurrence is similar over each surface type, where the relative occurrence of ice clouds decreases with increasing TCWV, which could be explained by more of the cloud condensate being allocated to liquid-water content.

AIRS tends to underestimate the relative occurrence of ice phase when compared with CCL in all but the wettest TCWV regime and the relative occurrence of liquid phase is more comparable across the TCWV regimes (Figs. 9c,d). Over open ocean, AIRS ice-phase relative occurrence increases with TCWV, which is opposite to CCL, and is underestimated relative to CCL by $\sim 40 \%$ in the driest TCWV regime. Over sea ice, the relationship between AIRS ice-phase relative occurrence and TCWV is like CCL, where ice-phase relative occurrence decreases with TCWV. For both surface types, AIRS overestimates liquid-phase relative occurrence anywhere from $5 \%$ to $20 \%$ depending on the TCWV regime. However, for each surface type, just like CCL, AIRS liquid-phase increases with TCWV. Over each surface type, AIRS unknown-phase relative occurrence is largest for all TCWV regimes except for $1-4 \mathrm{~kg} \mathrm{~m}^{-2}$ over sea ice. For open ocean, AIRS unknown is largest in the driest TCWV regime, which coincides with the peak of CCL mixed phase, and linearly decreases with increasing TCWV. For sea ice, AIRS unknown phase relative occurrence remains between $35 \%$ and $55 \%$ for all TCWV regimes, which corresponds to CCL mixed-phase having small variations across TCWV regimes.

\section{3) 1000-300-HPA TEMPERATURE DIFFERENCE COMPOSITE}

Figure 10 shows the frequency of occurrence of AIRS cloud phase and CCL cloud phase using all CCL detected cloud layers over sea ice and open ocean for $\Delta T_{1000-300}$ intervals ranging from 30 to $65 \mathrm{~K}$. Over open ocean, the $\Delta T_{1000-300}$ intervals most associated with cloudy AIRS and CCL FOVs are 50-55 K (Figs. 10a,c). Over sea ice, the $\Delta T_{1000-300}$ occurrence peak shifts to smaller values (Figs. 10b,d), which is consistent with colder surface conditions producing a more stable troposphere. 

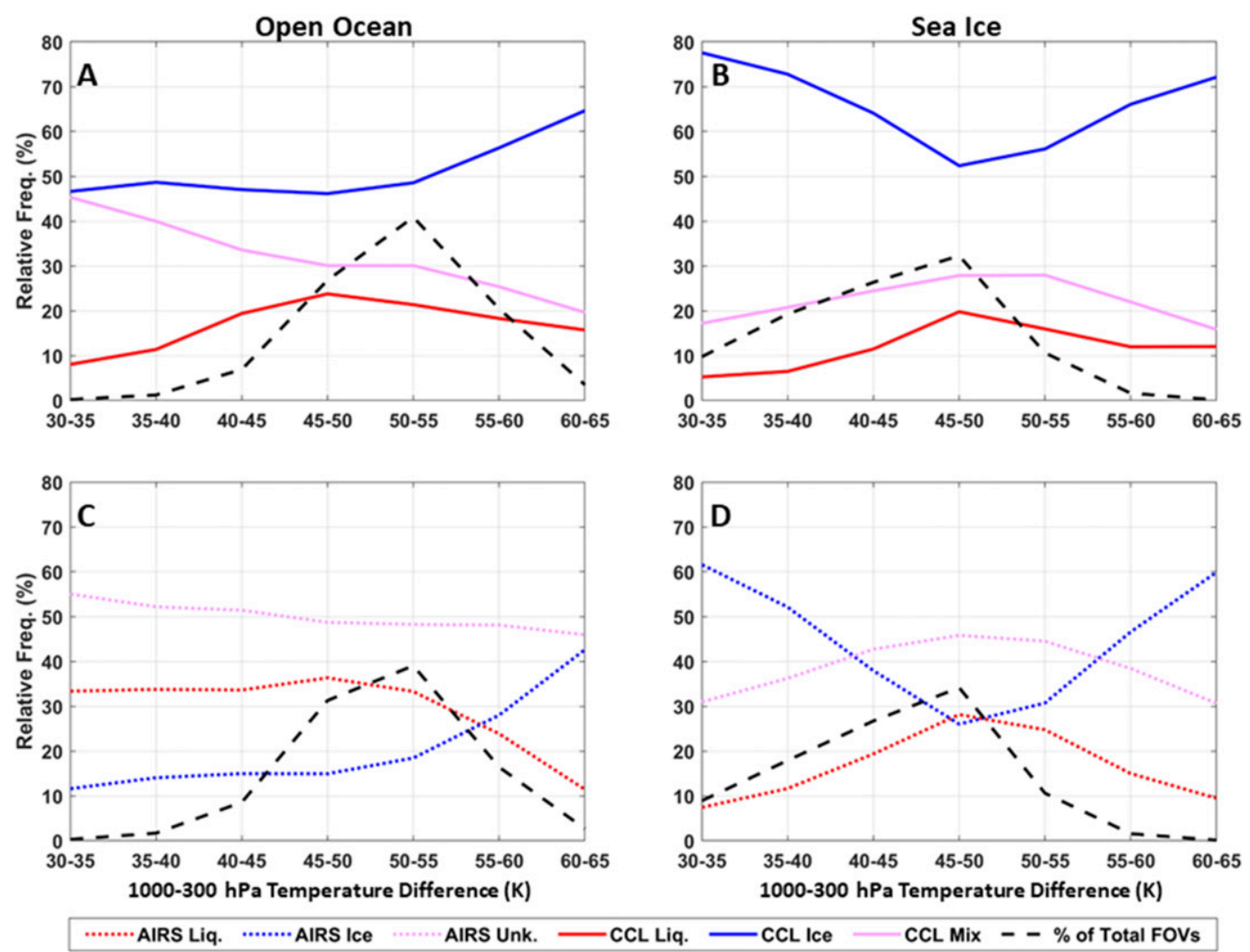

FIG. 10. (a) Frequency of occurrence of CCL cloud phase using all CCL detected cloud layers over open ocean for intervals of the temperature difference between 1000 and $300 \mathrm{hPa}\left(\Delta T_{1000-300}\right)$. The dotted black line represents the percentage of cloudy CCL FOVs that fall within a $\Delta T_{1000-300}$ interval. Normalization is with respect to all CCL FOVs with a cloud phase assigned in a given $\Delta T_{1000-300}$ interval. (b) As in (a), but for sea ice. (c) Frequency of occurrence of AIRS cloud phase over open ocean for intervals of $\Delta T_{1000-300}$. The dotted black line represents the percentage of cloudy AIRS FOVs that fall within a $\Delta T_{1000-300}$ interval. Normalization is with respect to all AIRS FOVs with a cloud phase assigned in a given $\Delta T_{1000-300}$ interval. (d) As in (c), but for sea ice.

Over each surface type, CCL liquid-phase relative occurrence shows a general increasing trend from $5 \%-$ $10 \%$ to $10 \%-15 \%$ with increasing $\Delta T_{1000-300}$. At higher $\Delta T_{1000-300}$, the Arctic troposphere can be more unstable and could thus favor the development of liquid cloud water. CCL mixed-phase cloud relative occurrence is largest in the smallest $\Delta T_{1000-300}$ regime (30-35 K) over open ocean and linearly decreases with increasing $\Delta T_{1000-300}$. Over sea ice, CCL mixed-phase relative occurrence varies from $15 \%$ to $30 \%$ and peaks between 50 and $55 \mathrm{~K}$. CCL ice-phase relative occurrence has a distinctly different relationship with $\Delta T_{1000-300}$ over each surface type. Over open ocean, it increases from $\sim 50 \%-60 \%$ with increasing $\Delta T_{1000-300}$, and over sea ice it decreases from $\sim 80 \%$ at the most stable $\Delta T_{1000-300}$ regimes, is minimum at $50 \%$ between 45 and $50 \mathrm{~K}$, and then linearly increases with increasing $\Delta T_{1000-300}$.

AIRS underestimates the relative occurrence of icephase over open ocean by $20 \%-40 \%$ when compared with CCL (Figs. 10a,c), but the relationship between ice-phase occurrence and $\Delta T_{1000-300}$ is similar to CCL. Over sea ice, AIRS and CCL agree that ice-phase occurrence is larger than liquid-phase across the $\Delta T_{1000-300}$ regimes and both AIRS and CCL's ice-phase relative occurrence have a very similar functional relationship with $\Delta T_{1000-300}$ (Figs. 10b,d). However, AIRS still underestimates ice-phase relative occurrence over sea ice by $10 \%-20 \%$. AIRS tends to overestimate the relative occurrence of liquid phase by $10 \%-20 \%$ over open ocean in comparison with CCL. Also, AIRS liquid-phase relative occurrence is largest in the smallest $\Delta T_{1000-300}$ regimes and smallest in the highest $\Delta T_{1000-300}$ regime, which is opposite to that of CCL over open ocean. Over sea ice, AIRS and CCL liquid-phase occurrence have a similar relationship with $\Delta T_{1000-300}$, but AIRS tends to overestimate liquid-phase relative occurrence by $5 \%-10 \%$ when compared with CCL. AIRS unknown-phase occurrence is larger than AIRS liquid and ice phase over all $\Delta T_{1000-300}$ regimes for open ocean and is not highly dependent on $\Delta T_{1000-300}$. 

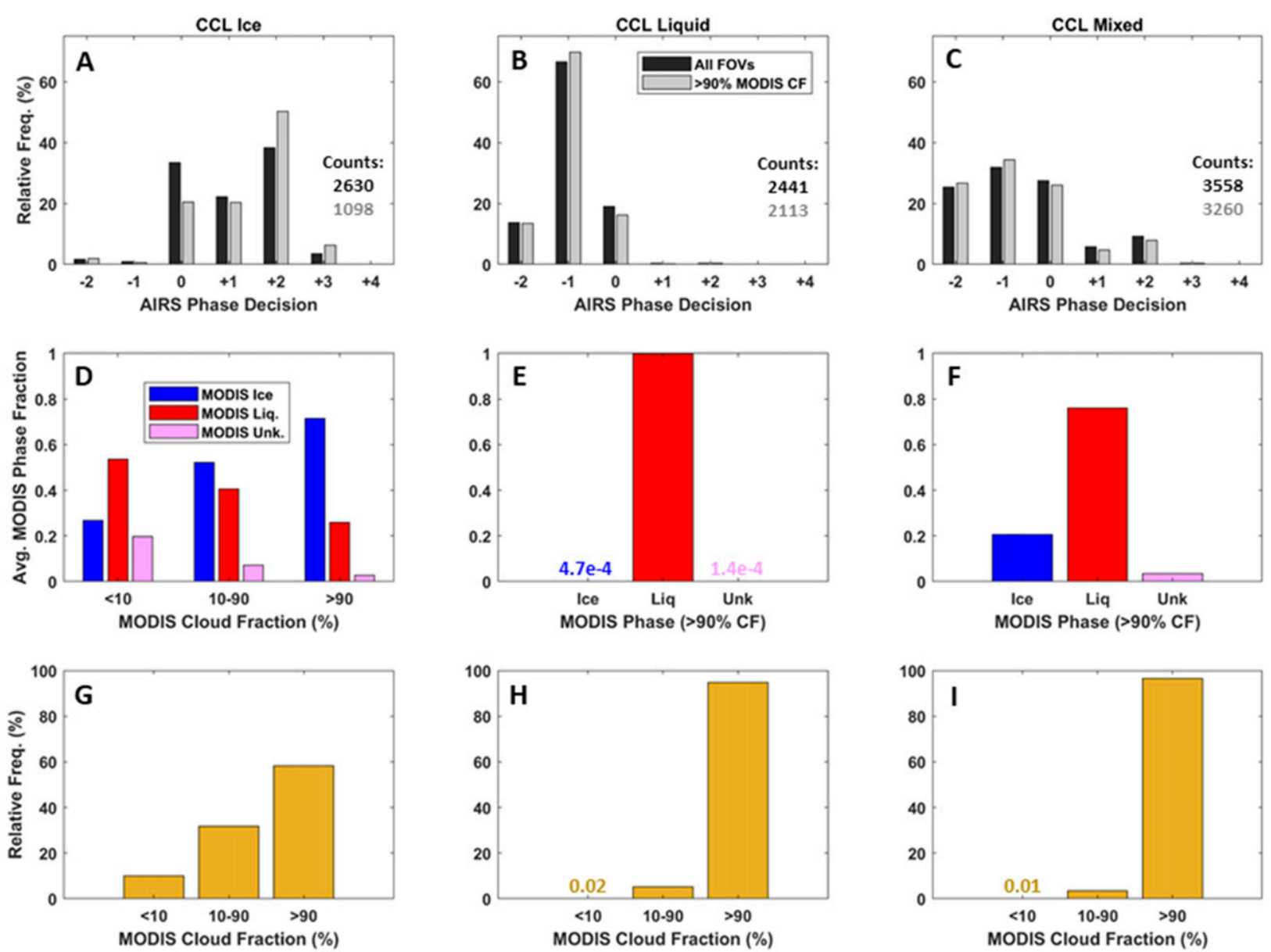

FIG. 11. For July 2009 over open ocean and sea ice for AIRS FOVs that contain single-layer-overcast homogenous (left) ice-, (center) liquid-, or (right) mixed-phase CCL FOV groups: (a)-(c) Frequency of occurrence of AIRS phase decisions for all AIRS FOVs collocated with CCL and MODIS (black) and for which the MODIS CF within the AIRS FOV is $>90 \%$ (gray). The counts represent the number of AIRS FOVs included for the "All FOVs" (black) and " $>90 \%$ MODIS CF" (gray) categories. The normalization is with respect to these counts. (d) Average MODIS cloud phase fraction (the number the MODIS pixels of a given phase divided by all MODIS pixels that are assigned a phase in the AIRS FOV and then averaged across all respective AIRS FOVs) for three intervals of MODIS CF. (e),(f) As in (d), but for $>90 \%$ MODIS CF only. (g)-(i) Frequency of occurrence of MODIS CF in three intervals. The normalization here is with respect to the number of all AIRS FOVs included in the analysis [count numbers in black in (a)-(c)].

Over sea ice, AIRS unknown phase is only greater than AIRS liquid and ice phase within the $40-55 \mathrm{~K}$ regimes.

The TCWV and $\Delta T_{1000-300}$ composite analyses indicate that AIRS and CCL disagree on the magnitude of relative occurrence of liquid and ice phases in the regimes. The AIRS unknown phase is a major contributor to such biases, especially over open ocean where CCL mixed-phase clouds are more prevalent. However, the relationship between AIRS cloud phase and TCWV and $\Delta T_{1000-300}$ regimes is similar to CCL, mainly over sea ice, and this indicates that AIRS cloud phase retrievals can be realistically used in Arctic cloud process studies. Last, it was determined that neither $\Delta T_{1000-300}$ nor TCWV regime significantly impacted AIRS phase classification skill.

\section{MODIS-AIRS-CCL case study}

A case study was conducted using daytime only collocated Arctic MODIS-AIRS-CCL FOVs for July 2009 in order to understand 1) the relationship between MODIS cloud amount and phase and AIRS Arctic cloud phase occurrence statistics and 2) the agreement between MODIS and CCL cloud phase classifications. MODIS cloud fraction (CF) within an AIRS FOV is defined as the ratio of MODIS pixels within an AIRS FOV that are flagged as probably or confident cloudy with respect to the total number of MODIS pixels in the AIRS FOV. Only AIRS FOVs with $>95 \%$ of MODIS pixels having a cloud mask decision are included.

Figures 11a-c show the occurrence frequency of AIRS cloud phase over open and sea ice together using all 
collocated MODIS-AIRS-CCL AIRS FOVs (black bars) and for those in which there is only greater than $90 \%$ MODIS CF in the AIRS FOVs (gray bars). This is shown for collocation with single-layer-overcast and homogenous CCL ice-phase groups (left column), CCL liquid-phase groups (middle column), and for CCL mixed-phase groups (right column). For each of the CCL phase groups (Figs. 11a-c), the frequency of occurrence of AIRS phase decisions is similar to what is observed for the entire 4-yr period. This indicates that the AIRS cloud phase classification skill determined in section 3 is robust to sampling differences. Figure 11 (middle row) shows the average MODIS cloud phase fraction (CPF) from AIRS FOVs that are collocated with each CCL cloud phase group (Figs. 11d-f). Here, MODIS CPF is defined as the ratio of MODIS pixels of a given phase relative to all MODIS pixels with a phase assigned in the AIRS FOV. This shown for three regimes of MODIS CF (CF $>10 \%, 10 \%<\mathrm{CF}<90 \%$, and $\mathrm{CF}>90 \%$ ) in Fig. 11d. For Figs. 11e and 11f, MODIS CF is greater than $90 \%$ only. Figures $11 \mathrm{~g}-\mathrm{i}$ show the frequency of occurrence of MODIS CF within three CF regimes. This is shown for collocation with each of the three CCL cloud phase groups (Figs. 11g-i).

MODIS CF occurrence in Figs. 11h and 11i indicates that most AIRS FOVs containing the CCL liquid and mixed-phase groups have greater than $90 \%$ cloud cover. Cloud amount in the Arctic reaches its peak in July and thus it is reasonable to observe a high occurrence of horizontal cloud coverage within these AIRS FOVs. For the AIRS FOVs with CCL liquid-phase groups, the average MODIS liquid CPF is $\sim 0.99$ (Fig. 11e). Marchant et al. (2016) shows that when evaluated with CALIPSO, MODIS correctly classifies liquid cloud scenes at a higher rate than ice cloud scenes, which could help explain the liquid-phase agreement between MODIS and CCL seen in this study. For the AIRS FOVs with CCL mixed-phase groups, average MODIS liquid and ice $\mathrm{CPF}$ is $\sim 0.75$ and $\sim 0.25$, respectively (Fig. 11f). This indicates that CCL mixed-phase clouds tend to correspond to MODIS liquid-phase classifications just as they correspond to AIRS liquid phase. For the AIRS FOVs with CCL ice groups, greater than $90 \%$ MODIS CF occurs about $60 \%$ of the time (Fig. 11g), and for such cases, the average MODIS ice and liquid CPF is $\sim 0.7$ and $\sim 0.3$, respectively.

When AIRS FOVs with less than 90\% MODIS CF are selected in the AIRS cloud phase evaluation for the CCL liquid and ice-phase groups, the amount of unknown-phase classifications increases. This corroborates the results of Kahn et al. (2011) and Guillaume et al. (2019), who presented a similar result for global AIRS observations. However, note that when $10 \%-90 \%$
MODIS CF is included in the AIRS phase evaluations for the CCL ice-phase groups the average ice and liquid MODIS CPF becomes comparable, and this effect can also be responsible for increasing AIRS unknown-phase classifications, as was shown in Kahn et al. (2011). Overall, MODIS cloud phase classification for ice and liquid scenes was consistent with CCL, which provides more confidence of the use of CCL for AIRS cloud phase evaluations during the 4-yr period.

\section{Conclusions}

In this study, AIRS Arctic cloud phase classification was evaluated against combined CloudSat-CALIPSO (CCL) observations over a 4-yr period. Our approach for this AIRS phase evaluation was distinctly different from the global evaluation performed by JN14, especially in our use of CCL mixed-phase classification. Overall, AIRS ice-phase classification skill in the Arctic for single-layer clouds is $>85 \%$ depending on the surface type, which is much higher than the classification skill for liquid phase. However, AIRS tends to classify more liquid-phase clouds as liquid phase in the Arctic relative to what was reported for the entire globe in JN14. It is possible that dry atmospheres lead to less ambiguous AIRS liquid phase classification, and this warrants future investigation. Mixed-phase clouds correspond to AIRS unknown and liquid phases. AIRS phase classification changes with horizontal scale of cloud phase mixing, thus the morphology of mixed-phase cloud systems could influence AIRS phase decisions. The results of the two-layer AIRS phase evaluation indicate that, in some cases, the upper-layer cloud is transparent enough in the IR for the lower cloud layer's phase signal to influence AIRS phase decisions. The two-layer systems produce mostly unknown AIRS phase classifications, and such systems should be considered when improving the AIRS cloud phase algorithm.

When using all collocated AIRS and CCL FOVS, AIRS ice-phase occurrence relative to the other AIRS phases was less than that for CCL ice-phase relative occurrence across latitudes, seasons, and TCWV and $\Delta T_{1000-300}$ regimes over both sea ice and open ocean. However, the liquid-phase relative occurrence had more agreement. This result likely is due to AIRS unknownphase classifications corresponding to CCL ice phase and mixed phase. The seasonal cycle, and in some cases, the dependence of cloud phase relative occurrence on TCWV and $\Delta T_{1000-300}$ regime, was similar between CCL and AIRS. This indicates that AIRS cloud phase data can capture the connections between the physical properties and processes in the Arctic atmosphere and cloud phase. Moreover, the accurate seasonal cycle of 
AIRS liquid and ice cloud phase indicates that AIRS could provide accurate occurrence anomalies on interseasonal to interannual scales.

The MODIS case study indicates that heterogeneous cloud cover and cloud phase distributions in the AIRS FOV correspond to increased unknown-phase classifications by AIRS, which verifies the findings of previous studies. Because MODIS cloud phase throughout the AIRS FOVs tended to correspond to cloud phase detected by CCL, and the case-study-based AIRS phase decision statistics were similar to that of the entire 4-yr period, the case study provides more confidence in the use of the CCL narrow track for AIRS phase evaluations.

Given that $42 \%$ of AIRS FOVs over the Arctic Ocean were classified as unknown phase by AIRS, it is pertinent to improve the algorithm. The results of this study both illuminate factors to consider for algorithm improvement and factors of secondary importance. For example, both TCWV and $\Delta T_{1000-300}$ did not significantly impact AIRS phase classification skill. However, surface type did correspond to $\sim 10 \%$ differences in AIRS phase classification skill for ice and liquid phase. Given that window channels are used in the AIRS phase algorithm, it is possible that differences in the surface emissivity of open ocean and sea ice may play a role in phase misclassifications for thin clouds. Channels that are less useful in moist lower latitudes could be used to improve cloud phase classifications in the dry Arctic. An information content analysis could shed light onto how cloud phase signatures influence AIRS channels in variable TCWV regimes. Mixed-phase cloud identification using the IR continues to be a challenge. Over the Arctic, AIRS frequently classifies mixed-phase clouds as either liquid or unknown and, according to the case study, MODIS frequently classifies them as liquid phase. However, because CCL can only identify the presence of liquid and ice within a single cloud layer, we could not further investigate how the influence of mixed-phase cloud vertical structure relates to AIRS or MODIS cloud phase decisions. This could be addressed by collocating ground-based high-spatial-resolution lidar measurements of Arctic mixed-phase clouds with AIRS and MODIS observations or generating synthetic radiances for cloud phase classification from large-eddy simulations that capture realistic mixed-phase cloud processes.

Acknowledgments. We thank two anonymous reviewers for their thoughtful and thorough reviews, which improved the presentation of the article. The research described in this paper was carried out at the University of Michigan and the Jet Propulsion Laboratory of the
California Institute of Technology under a contract with the National Aeronautics and Space Administration. Authors Yue, Fetzer, and Kahn were supported by NASA's Making Earth System Data Records for Use in Research Environments (MEASURES) program. Lead author Peterson was supported by the NASA FINESST program under Grant 80NSSC19K1331. The authors thank the support from the JPL AIRS project.

Data availability statement. The ERA5 data were obtained online (https://cds.climate.copernicus.eu), the 2BCloudClassLidar data were obtained online (http:// www.cloudsat.cira.colostate.edu/), the MODIS data were obtained through the Level-1 and Atmosphere Archive and Distribution System (LAADS; http:// ladsweb.nascom.nasa.gov/), and the AIRS/AMSU data were obtained from Goddard Earth Services Data and Information Services Center (http://daac.gsfc.nasa.gov/).

\section{REFERENCES}

Aumann, H. H., and Coauthors, 2003: AIRS/AMSU/HSB on the Aqua mission: Design, science objectives, data products, and processing systems. IEEE Trans. Geosci. Remote Sens., 41, 253-264, https://doi.org/10.1109/TGRS.2002.808356.

Baum, B. A., P. F. Soulen, K. I. Strabala, M. D. King, S. A. Ackerman, W. P. Menzel, and P. Yang, 2000: Remote sensing of cloud properties using MODIS Airborne Simulator imagery during SUCCESS. 2. Cloud thermodynamic phase. J. Geophys. Res., 105, 11 781-11 792, https://doi.org/10.1029/ 1999JD901090.

— - W. P. Menzel, R. A. Frey, D. C. Tobin, R. E. Holz, S. A. Ackerman, A. K. Heidinger, and P. Yang, 2012: MODIS cloud-top property refinements for Collection 6. J. Appl. Meteor. Climatol., 51, 1145-1163, https://doi.org/10.1175/ JAMC-D-11-0203.1.

Boisvert, L. N., and J. C. Stroeve, 2015: The Arctic is becoming warmer and wetter as revealed by the Atmospheric Infrared Sounder. Geophys. Res. Lett., 42, 4439-4446, https://doi.org/ 10.1002/2015GL063775.

Cavalieri, D. J., and C. L. Parkinson, 2012: Arctic sea ice variability and trends, 1979-2010. Cryosphere, 6, 881-889, https://doi.org/ 10.5194/tc-6-881-2012.

Cesana, G., J. E. Kay, H. Chepfer, J. M. English, and G. de Boer, 2012: Ubiquitous low-level liquid-containing Arctic clouds: New observations and climate model constraints from CALIPSOGOCCP. Geophys. Res. Lett., 39, L20804, https://doi.org/10.1029/ 2012GL053385.

Chahine, M. T., and Coauthors, 2006: Improving weather forecasting and providing new data on greenhouse gases. Bull. Amer. Meteor. Soc., 87, 911-926, https://doi.org/10.1175/BAMS87-7-911.

Cho, H. M., S. L. Nasiri, and P. Yang, 2009: Application of CALIOP measurements to the evaluation of cloud phase derived from MODIS infrared channels. J. Appl. Meteor. Climatol., 48, 21692180, https://doi.org/10.1175/2009JAMC2238.1.

Fetzer, E. J., 2012: A multi-sensor water vapor climate data record using cloud classification. NASA MEaSUREs Project, accessed 19 July 2020, https://disc.gsfc.nasa.gov/datasets? keywords $=$ Fetzer\&page $=1 \&$ project $=$ MEaSUREs . 
Goloub, P., M. Herman, H. Chepfer, J. Riedi, G. Brogniez, P. Couvert, and G. Seze, 2000: Cloud thermodynamical phase classification from the POLDER spaceborne instrument. J. Geophys. Res., 105, 14747-14 759, https://doi.org/10.1029/ 1999JD901183.

Guillaume, A., B. H. Kahn, E. J. Fetzer, Q. Yue, G. J. Manipon, B. D. Wilson, and H. Hua, 2019: Footprint-scale cloud type mixtures and their impacts on Atmospheric Infrared Sounder cloud property retrievals. Atmos. Meas. Tech., 12, 4361-4377, https://doi.org/10.5194/amt-12-4361-2019.

Hersbach H., and Coauthors, 2018: Operational global reanalysis: Progress, future directions and synergies with NWP. ERA Rep. Series 27, 65 pp., https://www.ecmwf.int/node/18765.

$\mathrm{Hu}, \mathrm{Y}$., and Coauthors, 2009: CALIPSO/CALIOP cloud phase discrimination algorithm. J. Atmos. Oceanic Technol., 26, 2293-2309, https://doi.org/10.1175/2009JTECHA1280.1.

Intrieri, J. M., C. W. Fairall, M. D. Shupe, P. O. G. Persson, E. L Andreas, P. S. Guest, and R. E. Moritz, 2002: An annual cycle of Arctic surface cloud forcing at SHEBA. J. Geophys. Res., 107, 8039, https://doi.org/10.1029/2000JC000439.

Jin, H. C., and S. L. Nasiri, 2014: Evaluation of AIRS cloudthermodynamic-phase determination with CALIPSO. J. Appl. Meteor. Climatol., 53, 1012-1027, https://doi.org/10.1175/JAMCD-13-0137.1.

Kahn, B. H., and Coauthors, 2008: Cloud type comparisons of AIRS, CloudSat, and CALIPSO cloud height and amount. Atmos. Chem. Phys., 8, 1231-1248, https://doi.org/10.5194/ acp-8-1231-2008.

, S. L. Nasiri, M. M. Schreier, and B. A. Baum, 2011: Impacts of sub-pixel cloud heterogeneity on infrared thermodynamic phase assessment. J. Geophys. Res., 116, D20201, https:// doi.org/10.1029/2011JD015774.

- and Coauthors, 2014: The Atmospheric Infrared Sounder version 6 cloud products. Atmos. Chem. Phys., 14, 399-426, https://doi.org/10.5194/acp-14-399-2014.

Kay, J. E., and T. L'Ecuyer, 2013: Observational constraints on Arctic Ocean clouds and radiative fluxes during the early $21 \mathrm{st}$ century. J. Geophys. Res. Atmos., 118, 7219-7236, https:// doi.org/10.1002/JGRD.50489.

Key, J., and J. Intrieri, 2000: Cloud particle phase determination with the AVHRR. J. Appl. Meteor., 39, 1797-1804, https:// doi.org/10.1175/1520-0450-39.10.1797.

Marchand, R., G. G. Mace, T. Ackerman, and G. Stephens, 2008: Hydrometeor detection using CloudSat-An Earth-orbiting 94-GHz cloud radar. J. Atmos. Oceanic Technol., 25, 519-533, https://doi.org/10.1175/2007JTECHA1006.1.

Marchant, B., S. Platnick, K. Meyer, G. T. Arnold, and J. Riedi, 2016: MODIS Collection 6 shortwave-derived cloud phase classification algorithm and comparisons with CALIOP. Atmos. Meas. Tech., 9, 1587-1599, https://doi.org/10.5194/amt-9-15872016.

Morrison, H., G. de Boer, G. Feingold, J. Harrington, M. D. Shupe, and K. Sulia, 2012: Resilience of persistent Arctic mixed-phase clouds. Nat. Geosci., 5, 11-17, https://doi.org/10.1038/ngeo1332.

Nasiri, S. L., and B. H. Kahn, 2008: Limitations of bispectral infrared cloud phase determination and potential for improvement. J. Appl. Meteor. Climatol., 47, 2895-2910, https://doi.org/ 10.1175/2008JAMC1879.1.

Peterson, C. A., X. Chen, Q. Yue, and X. Huang, 2019: The spectral dimension of Arctic outgoing longwave radiation and greenhouse efficiency trends from 2003 to 2016. J. Geophys. Res. Atmos., 124, 8467-8480, https://doi.org/10.1029/2019JD030428.

Platnick, S., and Coauthors, 2013: MODIS cloud optical properties: User guide for the Collection 6 level-2 MOD06/MYD06 product and associated level-3 datasets. MODIS MOD06 User Guide, 145 pp., https://modis-images.gsfc.nasa.gov/_docs/ C6MOD06OPUserGuide.pdf.

— , and Coauthors, 2017: The MODIS cloud optical and microphysical products: Collection 6 updates and examples from Terra and Aqua. IEEE Trans. Geosci. Remote Sens., 55, 502525, https://doi.org/10.1109/TGRS.2016.2610522.

Riedi, J., S. Platnick, B. A. Baum, F. Thieuleux, C. Oudard, F. Parol, J. M. Nicolas, and P. Dubuisson, 2010: Cloud thermodynamic phase inferred from merged POLDER and MODIS data. Atmos. Chem. Phys., 10, 11 851-11 865, https:// doi.org/10.5194/acp-10-11851-2010.

Sassen, K., Z. Wang, and D. Liu, 2008: Global distribution of cirrus clouds from CloudSat/Cloud-Aerosol Lidar and Infrared Pathfinder Satellite Observations (CALIPSO) measurements. J. Geophys. Res., 113, D00A12, https://doi.org/ 10.1029/2008JD009972.

Schreier, M. M., B. H. Kahn, A. Eldering, D. A. Elliott, E. Fishbein, F. W. Irion, and T. S. Pagano, 2010: Radiance comparisons of MODIS and AIRS using spatial response information. J. Atmos. Oceanic Technol., 27, 1331-1342, https:// doi.org/10.1175/2010JTECHA1424.1.

Shupe, M. D., 2011: Clouds at Arctic atmospheric observatories. Part II: Thermodynamic phase characteristics. J. Appl. Meteor. Climatol., 50, 645-661, https://doi.org/10.1175/2010JAMC2468.1. , and J. M. Intrieri, 2004: Cloud radiative forcing of the Arctic surface: The influence of cloud properties, surface albedo, and solar zenith angle. J. Climate, 17, 616-628, https://doi.org/ 10.1175/1520-0442(2004)017<0616:CRFOTA > 2.0.CO;2.

Tanelli, S., S. L. Durden, E. Im, K. S. Pak, D. G. Reinke, P. Partain, J. M. Haynes, and R. T. Marchand, 2008: CloudSat's cloud profiling radar after two years in orbit: Performance, calibration, and processing. IEEE Trans. Geosci. Remote Sens., 46, 3560-3573, https://doi.org/10.1109/TGRS.2008.2002030.

Thompson, D. R., B. H. Kahn, R. O. Green, S. A. Chien, E. M. Middleton, and D. Q. Tran, 2018: Global spectroscopic survey of cloud thermodynamic phase at high spatial resolution, 2005-2015. Atmos. Meas. Tech., 11, 1019-1030, https://doi.org/ 10.5194/amt-11-1019-2018.

Wang, T., E. J. Fetzer, S. Wong, B. H. Kahn, and Q. Yue, 2016: Validation of MODIS cloud mask and multilayer flag using CloudSat-CALIPSO cloud profiles and a cross-reference of their cloud classifications. J. Geophys. Res. Atmos., 121, 11 620-11 635, https://doi.org/10.1002/2016JD025239.

Wang, Z., D. Vane, G. Stephens, and D. Reinke, 2013: CloudSat Project: Level 2 combined radar and lidar cloud scenario classification product process description and interface control document. California Institute of Technology, Jet Propulsion Laboratory Doc., 61 pp., http://www.cloudsat.cira.colostate.edu/ sites/default/files/products/files/2B-CLDCLASS-LIDAR_PDICD.P_ R04.20120522.pdf.

Yue, Q., B. H. Kahn, E. J. Fetzer, and J. Teixeira, 2011: Relationship between marine boundary layer clouds and lower tropospheric stability observed by AIRS, CloudSat and CALIOP. J. Geophys. Res., 116, D18212, https://doi.org/10.1029/2011JD016136. 